\title{
Triple arrays and Youden squares
}

\author{
Tomas Nilson · Lars-Daniel Öhman
}

Received: 23 September 2013 / Revised: 15 January 2014 / Accepted: 17 January 2014 /

Published online: 4 February 2014

C) Springer Science+Business Media New York 2014

\begin{abstract}
This paper addresses the question of when triple arrays can be constructed from Youden squares by removing a column together with the symbols therein, and then exchanging the role of columns and symbols. The scope of the investigation is limited to the standard case of triple arrays with $v=r+c-1$. For triple arrays with $\lambda_{c c}=1$ it is proven that they can never be constructed in this way, and for triple arrays with $\lambda_{c c}=2$ it is proven that there always exists a suitable Youden square and a suitable column for this construction. Further, it is proven that Youden square constructed from a certain family of difference sets never give rise to triple arrays in this way but always gives rise to double arrays. Finally, it is proven that all triple arrays in the single known infinite family, the Paley triple arrays, can all be constructed in this way for some suitable choice of Youden square and column.
\end{abstract}

Keywords Triple array $\cdot$ Double array $\cdot$ Youden square $\cdot$ Difference set $\cdot$ SBIBD

Mathematics Subject Classification $05 \mathrm{~B} 05 \cdot 05 \mathrm{~B} 10 \cdot 05 \mathrm{~B} 30$

\section{Introduction}

We will assume familiarity with the standard definitions of balanced incomplete block designs (BIBD's) and symmetric balanced incomplete block designs (SBIBD's). A triple array is an $r \times c$ array on $v$ symbols arranged so that no symbol occurs more than once in any row or column, that satisfies the following four conditions:

Communicated by C. J. Colbourn.

T. Nilson

Department of Science Education and Mathematics, Mid-Sweden University, Sundsvall, Sweden e-mail: tomas.nilson@miun.se

L.-D. Öhman $(\varangle)$

Department of Mathematics and Mathematical Statistics, Umeå University, Umeå, Sweden

e-mail: lars-daniel.ohman@math.umu.se 
TA1. Each symbol occurs $k$ times (equireplicate).

TA2. Any two distinct rows contain $\lambda_{r r}$ common symbols.

TA3. Any two distinct columns contain $\lambda_{c c}$ common symbols.

TA4. Any row and column contain $\lambda_{r c}$ common symbols.

For a general triple array with the above parameters, we will use the notation $T A\left(v, k, \lambda_{r r}, \lambda_{c c}, \lambda_{r c}: r \times c\right)$. An array as above that satisfies conditions TA1-TA3 is called a double array, for which we use the notation $D A\left(v, k, \lambda_{r r}, \lambda_{c c}: r \times c\right)$. Thus any triple array is a fortiori a double array, and we shall call a double array that does not satisfy TA4 a proper double array.

If we exchange the roles of columns and symbols in a triple array, we get an incomplete $r \times v$ array on $c$ symbols, with no repetitions in any row or column, satisfying the following four conditions, corresponding to the four above conditions TA1-TA4:

RTA1. Every column has $k$ occupied cells.

RTA2. For any pair of rows, there are $\lambda_{r r}$ columns in which both rows are occupied.

RTA3. Every pair of symbols must occur together in $\lambda_{c c}$ columns.

RTA4. The columns with occupied cells in row $i$ must contain every symbol exactly $\lambda_{r c}$ times, for all $i, 1 \leq i \leq r$.

We will call this the $R L$ form of a triple array (or, correspondingly, a double array). We will in some instances use the notation $T_{R L}$ for the $R L$ form of a triple array $T$.

Agrawal [1] studied what he called designs for two-way elimination of heterogeneity, a class of designs which includes triple arrays as a subset. Agrawal proposed a construction of triple arrays which relied on a certain rearranging step. However, Agrawal could not prove that this step could always be carried out. Subsequently, Raghavarao and Nageswararao [6] claimed to have proven, using a distinct representatives argument, that the rearranging step could in fact always be carried out, but, as noted by Wallis and Yucas [7], the proof is flawed.

In a concluding remark, Raghavarao and Nageswararao also claim ${ }^{1}$ that taking an arbitrary Youden square with suitable parameters and deleting an arbitrary column together with its symbols always produces the $R L$ form of a triple array. We recall the definition of a Youden square:

Definition 1 An $r \times v$ array, with $1<r<v$ on the symbols $1, \ldots, v$ is a Youden square if the following conditions are met.

YS1. Every symbol appears exactly once in each row.

YS2. Every symbol appears at most once in each column.

YS3. Every pair of columns intersects in a constant number of symbols.

Note that if we take the columns of a Youden square to be the blocks of a BIBD, $D$, then $D$ is a SBIBD, and each pair of symbols occurs in the same number of blocks (cf. [2, Proposition 2.4.8]). Also, by a well-known construction using sets of distinct representatives, the elements in the blocks of any SBIBD may be rearranged so as to form a Youden square.

For ease of reference, we state Raghavarao's and Nageswararao's proposed construction as

Construction 1 Let $Y$ be a Youden square, and $C$ a column in $Y$. Let $R$ be produced from $Y$ by deleting $C$ from $Y$ together with all the symbols in $C$.

1 This idea seems to originate from an anonymous referee of Raghavarao's and Nageswararao's paper. 


$\begin{array}{rrrrrrrrrrr}\mathbf{1} & \mathbf{2} & \mathbf{3} & \mathbf{4} & \mathbf{5} & 6 & 7 & 8 & 9 & 10 & 11 \\ \mathbf{2} & 7 & \mathbf{4} & \mathbf{5} & 9 & 11 & \mathbf{1} & 6 & \mathbf{3} & 8 & 10 \\ \mathbf{3} & \mathbf{4} & 6 & 9 & \mathbf{2} & 7 & 8 & 10 & 11 & \mathbf{5} & \mathbf{1} \\ \mathbf{4} & 8 & 7 & 6 & 10 & \mathbf{5} & \mathbf{3} & \mathbf{1} & \mathbf{2} & 11 & 9 \\ \mathbf{5} & 11 & 10 & 8 & 7 & \mathbf{1} & 9 & \mathbf{2} & 6 & \mathbf{3} & \mathbf{4}\end{array}$

Fig. 1 A Youden square $Y_{1}$ where the deletion of the first column and the symbols therein does not produce the $R L$ form of a triple array

$\begin{array}{rrrrrrrrrrr}1 & - & 3 & 4 & - & 6 & - & 8 & - & - & 11 \\ - & - & 4 & - & - & 11 & 1 & 6 & 3 & 8 & - \\ 3 & 4 & 6 & - & - & - & 8 & - & 11 & - & 1 \\ 4 & 8 & - & 6 & - & - & 3 & 1 & - & 11 & - \\ - & 11 & - & 8 & - & 1 & - & - & 6 & 3 & 4\end{array}$

Fig. 2 The Youden square $Y_{1}$ with the 5 th column and the symbols therein deleted, producing the $R L$ form of a double array

$\begin{array}{rrrrrrrrrrr}1 & 2 & 3 & 4 & 5 & 6 & 7 & 8 & 9 & 10 & 11 \\ 2 & 1 & 8 & 6 & 7 & 3 & 4 & 5 & 10 & 11 & 9 \\ 3 & 11 & 1 & 7 & 9 & 2 & 8 & 10 & 4 & 5 & 6 \\ 4 & 10 & 11 & 1 & 8 & 9 & 2 & 6 & 3 & 7 & 5 \\ 5 & 9 & 6 & 10 & 1 & 7 & 11 & 2 & 8 & 3 & 4\end{array}$

\begin{tabular}{rrrrrrrrrr|rrrrrr}
- & - & - & - & 6 & 7 & 8 & 9 & 10 & 11 & 6 & 7 & 8 & 9 & 10 & 11 \\
- & 8 & 6 & 7 & - & - & - & 10 & 11 & 9 & 5 & 6 & 7 & 8 & 9 & 10 \\
11 & - & 7 & 9 & - & 8 & 10 & - & - & 6 & 4 & 2 & 10 & 8 & 9 \\
10 & 11 & - & 8 & 9 & - & 6 & - & 7 & - & 7 & 9 & 4 & 5 & 1 & 2 \\
9 & 6 & 10 & - & 7 & 11 & - & 8 & - & - & 2 & 5 & 8 & 1 & 3 & 6
\end{tabular}

Fig. 3 A Youden square where the deletion of the first column and the symbols therein produces the $R L$ form of a triple array

We will sometimes say that $R$ is constructed from $Y$ modulo $C$. If the $R L$ form of the triple array $T$ can be constructed from the Youden square $Y$ through Construction 1, we will say that $Y$ is compatible with $T$. Again, as pointed out by Wallis and Yucas using the $5 \times 11$ counterexample in Fig. 1 (here we have rearranged the columns for normalization), it is not always true that this construction yields the $R L$ form of a triple array. In fact, for the example in Fig. 1, neither RTA2 nor RTA4 are satisfied if $C$ is taken to be the first column. For instance, columns 6 through 11 are occupied in the first row, and these columns contain the symbol 6 three times and the symbol 7 two times, thus violating RTA4, and rows 1 and 2 share four columns, whereas rows 1 and 4 share two columns, thus violating RTA2.

No matter which column is deleted, the resulting array is not the $R L$ form of a triple array. Note, however, that deleting column 5 or 10 does produce the $R L$ form of a double array, as in Fig. 2. To see that this is not a triple array, observe that the columns with occupied cells in row 1 contain the symbol 1 four times, and the symbol 3 two times, thus violating RTA4. This raises the question of whether Construction 1 will always give the $R L$ form of a double array for some choice of column.

Also, using the $5 \times 11$ Youden square in Fig. 3 (top of figure) and deleting the first column together with its symbols produces the $R L$ form (bottom left) of a triple array (bottom right). This raises the question of whether Construction 1 will always give a triple array for some Youden square with the desired parameters and some choice of column.

In this paper, we investigate this possible connection between Youden squares and triple arrays. The rest of the paper is structured as follows: In Sect. 2 we introduce some useful 
definitions and results. In Sect. 3 we prove that Youden squares with parameters corresponding to projective planes can never be used in Construction 1 to produce the $R L$ form of a triple array, but that they always give rise to double arrays, regardless of the choice of column. We also prove that for all triple arrays $T$ with $\lambda_{c c}=2$, one can find a Youden square $Y$ such that employing Construction 1 on $Y$ modulo a suitable choice of column will give the $R L$ form of $T$. In Sect. 4 we prove that $(q-1) / 2 \times q$ Youden squares constructed from certain difference sets in a finite field of order $q$, where $q \equiv 3(\bmod 4)$ is a prime power can never give rise to triple arrays through Construction 1, regardless of the choice of column, but that they always yield double arrays. Section 5 is devoted to showing that so-called Paley triple arrays (which, incidentally, is the only known infinite family of triple arrays) can all be constructed using Construction 1. Section 6 concludes.

\section{Preliminaries}

We recall the definition of a residual design.

Definition 2 Let $\mathcal{D}=(X, \mathcal{B})$ be an SBIBD and let $B \in \mathcal{B}$. The residual design of $\mathcal{D}$ with respect to $B$, denoted $\mathcal{D}^{B}$, has point set $X \backslash B$ and the blocks are the sets $B_{i} \backslash B$ for $B_{i} \in \mathcal{B} \backslash\{B\}$.

As indicated by the terminology, a residual design is in fact a BIBD. We state this wellknown fact as a proposition.

Proposition 1 Let $\mathcal{D}$ be $a(v, k, \lambda)-S B I B D$ and let $B$ be a block of $\mathcal{D}$. Then $\mathcal{D}^{B}$ is a $(v-k, v-1, k, k-\lambda, \lambda)-B I B D$.

We can now establish that an array obtained from any Youden square $Y$ by removing an arbitrary column $C$ together with its symbols (that is, by applying Construction 1 to $Y$ modulo $C$ ) always satisfies conditions RTA1 and RTA3.

Proposition 2 Let $Y$ be a Youden square, and $C$ be a column in $Y$. Let $R$ be constructed from $Y$ modulo $C$ using Construction 1. Then $R$ satisfies conditions RTA1 and RTA3.

Proof Since the columns of $Y$ form the blocks of a SBIBD, the columns of $R$ form the residual design with respect to the removed column. By Proposition 1, $R$ is a BIBD, and hence each column has the same number of occupied cells (the block size in the BIBD), so RTA1 holds. Similarly, RTA3 holds since this is true already in $Y$.

Moving on to triple arrays, we will use the following two lemmas, which can be found in [3] and [4], respectively.

Lemma 1 Suppose $T$ is a $T A\left(v, k, \lambda_{r r}, \lambda_{c c}, \lambda_{r c}: r \times c\right)$ with $v=r+c-1$. Then $\lambda_{c c}=$ $r-\lambda_{r c}=r-k$.

Lemma 2 Suppose $T$ is a $T A\left(v, k, \lambda_{r r}, \lambda_{c c}, \lambda_{r c}: r \times c\right)$ with $v=r+c-1$. Then $k+\lambda_{r r}=c$ and $k+\lambda_{c c}=r$.

We now prove some preliminary results concerning the structure of triple arrays.

Lemma 3 Suppose $T$ is a $T A\left(v, k, \lambda_{r r}, \lambda_{c c}, \lambda_{r c}: r \times c\right)$ with $v=r+c-1$. Then in any pair of rows, exactly $\lambda_{c c}-1$ symbols are missing. 
Proof The number of distinct symbols in use in two distinct rows is $\lambda_{r r}+2 k$, since they have $\lambda_{r r}$ symbols in common, and by Lemma 2, there are $k=c-\lambda_{r r}$ distinct symbols in each of the two rows. Since $v=r+c-1$, we have

$$
\begin{aligned}
v-r=c-1 & \Longleftrightarrow v-(r-k)=c+k-1 \\
& \Longleftrightarrow v-\lambda_{c c}=\lambda_{r r}+2 k-1,
\end{aligned}
$$

from which it follows that $\lambda_{c c}-1$ symbols are missing, as there are $v$ symbols in total.

Note that Lemma 3 is not true for the single known triple array with $v>r+c-1$, namely the $T A(35,3,5,1,3: 7 \times 15)$ presented in [3]. If we reformulate Lemma 3 in terms of the $R L$ form, we get Lemma 4.

Lemma 4 The empty cells in the RL form of a triple array with $v=r+c-1$ are distributed so that every set of 2 rows are empty in exactly $\lambda_{c c}-1$ common columns.

We will also need information about the distribution of symbols in the $R L$ form of a triple array.

Lemma 5 The symbols in the RL form of a triple array are distributed so that the set of columns with empty cells in row $i$ contains each symbol exactly $r-k$ times.

Proof Note that any symbol $\sigma$ must be used exactly $r$ times in the $R L$ form. Now, observe that there are $v-c$ empty cells in row $i$, for any $i$. Thus, by RTA1, there are $k(v-c)=$ $r c-k c=c(r-k)$ filled cells in the columns with empty cells in row $i$. In these cells, a set of $c$ symbols are distributed.

Suppose, for a contradiction, that some symbol $\sigma$ is used at most $(r-k)-1$ times in this set of $v-k$ columns. Then $\sigma$ must be used at least $r-(r-k)+1=k+1$ times in the remaining $k$ columns. This, however, is impossible, since each symbol can be used at most once in any column.

Conversely, suppose that some symbol $\sigma$ is used at least $(r-k)+1$ times. Then some other symbol will be used at most $(r-k)-1$ times, and again we have a contradiction.

If $v=r+c-1$, then by Lemma 1 we may write $r-k=\lambda_{c c}$ in Lemma 5.

Definition 3 Let $A$ be an equireplicate array (i.e. satisfying TA1) with replication number $k$ and with no repeated elements in any row or column. Let $x \neq y$ be two distinct symbols in $A$. Suppose that $\{x, y\}$ occurs in $r_{1}$ rows and in $c_{1}$ columns of $A$, and let $\mu(x, y)=r_{1}+c_{1}$. If $\mu(x, y)=\mu$ is constant over all pairs $\{x, y\}$, we say that $A$ is a balanced grid. We write $B G(v, k, \mu: r \times c)$ for an $r \times c$ balanced grid with $v$ points.

McSorley [4] established the following correspondence between triple arrays and balanced grids.

Theorem 2 When $v=r+c-1$ every triple array is a $T A(v, k, c-k, r-k, k: r \times c)$, and every balanced grid is a $B G(v, k, k: r \times c)$, and they are equivalent.

If we translate the balanced grid property to the $R L$ form of the corresponding triple array, we have the following corollary.

Corollary 1 For any two columns $C_{1}$ and $C_{2}$ in the RL form of a triple array with $v=$ $r+c-1$, the sum of the number of common rows and the number of common symbols of $C_{1}$ and $C_{2}$ is constant, $k$. 


\section{Constructing triple arrays from Youden squares}

We now turn to the question of whether all Youden squares give rise to the $R L$ form of some double array or triple array, at least for a suitable choice of column. It is natural to begin this line of inquiry by considering some well-known families of SBIBD's, and the simplest case is those SBIBD's that might produce triple arrays $T$ with $v=r+c-1$ and $\lambda_{c c}=1$.

\subsection{Youden squares corresponding to projective planes}

We apply Construction 1 with the particular parameters of a projective plane. The existence of a projective plane of order $q$ follows from the well-known construction from a finite field of order $q$, but the following theorem works for arbitrary projective planes.

Theorem 3 Construction 1 applied to a Youden square $Y$ corresponding to a projective plane of order $q$, that is a $\left(q^{2}+q+1, q+1,1\right)-S B I B D$, yields the $R L$ form $R$ of a proper double array, regardless of the choice of column.

Proof Let $B_{j}$ denote the support of the column $C_{j}$ in $Y$, and let $V$ denote the set of symbols of $Y$. Let $B_{0}$ denote the removed column. We first observe that no entry is repeated in any row or column of $R$, since no entry is repeated in any row or column of $Y$. Since every symbol of $V$ occurs exactly once in each row of $Y$, it follows that each symbol in $V \backslash B_{0}$ occurs exactly once in each row of $R$. Note that $\left|V \backslash B_{0}\right|=q^{2}$. Conditions RTA1 and RTA3 are satisfied by Proposition 2.

We now prove that condition RTA2 is satisfied. Two rows of $R$ cannot have empty cells in the same column $j$, as this would mean that two elements of $B_{0}$ would meet both in $B_{0}$ and in another block $B_{j}, j \neq 0$. This is impossible, since the columns of $Y$ form a SBIBD $D$, and any two blocks of $D$ intersect in exactly one element. This means that any two rows of $R$ have $q^{2}-q$ occupied columns in common, and thus RTA2 holds.

We now prove that condition RTA4 is never satisfied. Fix a row $i$ in $R$, and consider the subarray $E$ of $R$ that consists of the $q$ columns that have no occupied cells in row $i$. Any two columns in $E$ intersect in exactly one symbol, and these symbols are distinct for distinct pairs of columns. Therefore $\frac{q(q-1)}{2}$ of the symbols in $E$ occur twice, while the other $q^{2}-2 \frac{q(q-1)}{2}=q$ symbols in $E$ occur only once.

Since every symbol occurs $q+1$ times in $R$, it follows that among the columns that have occupied cells in row $i, \frac{q(q-1)}{2}$ of the symbols occur in these columns $q-1$ times, $q$ of the symbols occur $q$ times and the remaining $\frac{q(q+1)}{2}$ symbols occur $q+1$ times. Hence, $R$ does not satisfy RTA4.

Since any SBIBD with parameters $\left(q^{2}+q+1, q+1,1\right)$ is a projective plane, and the columns of any Youden square form the blocks of a SBIBD, we see that Construction 1 can never be used to produce a triple array with parameters $\left(q^{2}+q, q, q^{2}-q, 1, q:(q+1) \times q^{2}\right)$, regardless of the choice of Youden square or column. If we assume that $v=r+c-1$, and set $k=q$, it follows from Lemmas 1 and 2 that any triple array with $\lambda_{c c}=1$ is a $T A\left(q^{2}+q, q, q^{2}-q, 1, q:(q+1) \times q^{2}\right)$, so in fact no triple array with $v=r+c-1$ and $\lambda_{c c}=1$ can be produced using this method. We formulate this conclusion as a theorem.

Theorem 4 Let $T$ be a triple array with $\lambda_{c c}=1$ and $v=r+c-1$. Then there is no Youden square compatible with $T$. 
3.2 A proposed general method for constructing compatible Youden squares from triple arrays

Consider the following proposed construction of Youden squares from triple arrays. This type of construction, where a Youden square is produced from the $R L$ form of a triple array, will be referred to as an extension.

Construction 5 Let $T=T A\left(v, k, \lambda_{r r}, \lambda_{c c}, \lambda_{r c}: r \times c\right)$ be a triple array with $v=r+c-1$, and $T_{R L}$ be the corresponding $R L$ form. Let $Y$ be an array constructed from $T_{R L}$ by the following steps.

- Add a column $C_{0}$ to $T_{R L}$ with $r$ new symbols, $s_{1}, s_{2}, \ldots, s_{r}$, in this order (the addition step).

- Suppose the empty cells in column $C_{j}$ in $T_{R L}$ lie in rows $i_{1}, i_{2}, \ldots, i_{\lambda_{c c}}$. Then use the symbols $s_{i_{1}}, s_{i_{2}}, \ldots, s_{i_{\lambda_{c c}}}$ to fill the empty cells in column $C_{j}$, in such a way that no symbol is repeated in any row (the completion step).

For Construction 5 to make sense, note first that by RTA1, there are exactly $r-k=\lambda_{c c}$ empty cells in each column of $T_{R L}$. If the construction can be carried out, $Y$ is trivially compatible with $T$.

In Sect. 5, we will give a construction of a compatible Youden square where the set of symbols used to fill the empty rows $i_{1}, i_{2}, \ldots, i_{\lambda_{c c}}$ in a column is not the set $s_{i_{1}}, s_{i_{2}}, \ldots, s_{i_{\lambda c c}}$, so there are, in general, other ways of carrying out the completion step.

The completion step of Construction 5 is clearly not trivial to carry out, but the only difficulty that needs to be handled is for there to be no repeated symbols in any row. If this is achieved, the other Youden square properties will follow. We formulate this as a proposition.

Proposition 3 Let $T$ be a triple array with $v=r+c-1$ and let $T_{R L}$ the corresponding $R L$ form. Suppose $Y$ is produced from $T_{R L}$ by means of Construction 5, so that there is no repeated symbol in any row. Then $Y$ is a Youden square.

Proof Property YS1 follows from the fact that all cells are filled, and the assumption that there are no repeated symbols in any row. Property YS2 follows by construction and the fact that there are no repeated symbols in any column of $T_{R L}$.

To see that property YS3 holds, note first that obviously column $C_{0}$ intersects any other column in exactly $\lambda_{c c}$ symbols. Let $r_{c}(i, j)$ and $s_{c}(i, j)$ denote the number of common rows of columns $C_{i}$ and $C_{j}$, and the number of common symbols of columns $C_{i}$ and $C_{j}$, respectively. By construction, the number of new common symbols of columns $C_{i}$ and $C_{j}$ added in the completion step is equal to the number of cells that are empty in both $C_{i}$ and $C_{j}$. We denote this quantity by $s_{c}^{\prime}(i, j)$. We will prove that $s_{c}(i, j)+s_{c}^{\prime}(i, j)=\lambda_{c c}$, which implies that YS3 holds.

The number of rows that are empty in both columns $C_{i}$ and $C_{j}$ equals $s_{c}^{\prime}(i, j)$, by construction. The number of rows that are empty in exactly one of the two columns is $2\left(k-r_{c}(i, j)\right)$, since there are $k$ filled cells in each column and they share $r_{c}(i, j)$ filled rows. We may therefore write

$$
r=s_{c}^{\prime}(i, j)+2\left(k-r_{c}(i, j)\right)+r_{c}(i, j)=s_{c}^{\prime}(i, j)+2 k-r_{c}(i, j) .
$$

By Corollary $1, r_{c}(i, j)+s_{c}(i, j)=k$ for all distinct $i, j$ with $1 \leq i, j \leq v$. We may therefore rewrite this equation as

$$
r-k=s_{c}^{\prime}(i, j)+k-r_{c}(i, j)=s_{c}^{\prime}(i, j)+s_{c}(i, j)
$$


By Lemma $1, \lambda_{c c}=r-k$, so it follows that $\lambda_{c c}=s_{c}^{\prime}(i, j)+s_{c}(i, j)$, which concludes the proof.

We would like to propose the following conjecture, for which we will present further evidence below. The case $\lambda_{c c}=1$ is excluded by Theorem 3, and therefore, if true, Conjecture 1 is sharp with regard to the condition on $\lambda_{c c}$.

Conjecture 1 Let $T$ be a triple array with $\lambda_{c c} \geq 2$. Then Construction 5 can be used to produce a Youden square $Y$ that will produce the $R L$ form of $T$ modulo column $C_{0}$. In particular, the completion step can always be carried out.

In the next section, we will prove this conjecture in the case $\lambda_{c c}=2$. We have also verified the conjecture for the $T A(14,4,4,3,4: 7 \times 8)$, the $T A(24,6,10,3,6: 9 \times 16)$, the $T A(30,7,14,3,6: 10 \times 21)$, and the $T A(44,9,24,3,9: 12 \times 33)$ presented in [3], all with $\lambda_{c c}=3$.

Note also that the $T A(14,4,4,3,4: 7 \times 8)$ is a so-called Paley triple array, and that we prove below, in Theorem 10, that for all such triple arrays $T$, there exists a Youden square $Y$ and a column $C$ such that the $R L$ form of $T$ can be produced from $Y$ by applying Construction 1 modulo $C$.

\subsection{Youden squares corresponding to biplanes}

In this section, we will show that for any given triple array $T$ with $v=r+c-1$ and $\lambda_{c c}=2$ one can find a Youden square (which corresponds to a biplane, that is a SBIBD with parameters $(v+1, r, 2))$, that can produce $T$ through Construction 1 . The completion step in this case is detailed in Construction 6, and is a special case of Construction 5.

Construction 6 Let $T=T A\left(v, k, \lambda_{r r}, 2, \lambda_{r c}: r \times c\right)$ be a triple array with $v=r+c-1$, and $T_{R L}$ be the corresponding $R L$ form. Let $Y$ be the array constructed from $T_{R L}$ by the following steps.

- Add a column $C_{0}$ to $T_{R L}$ with $r$ new symbols, $s_{1}, s_{2}, \ldots, s_{r}$, in this order.

- For any empty cell $c_{i} \in T_{R L}$, set $s\left(c_{i}\right)=s_{j}$ iff the unique other empty cell in the same column as $c_{i}$ lies in row $j$.

Example 1 Let $T_{5}$ be the $5 \times 6$ triple array in Fig. 4. Its $R L$ form has two empty cells in each column. If these empty cells are filled in accordance with Construction 6, using symbols $\{7,8,9,10,11\}$, the result is the Youden square in Fig. 5.

\begin{tabular}{rrrrrr|rrrrrrrrrr}
1 & 2 & 3 & 4 & 5 & 6 & 1 & 2 & 3 & 4 & 5 & 6 & - & - & - & - \\
4 & 7 & 1 & 3 & 8 & 9 & 3 & - & 4 & 1 & - & - & 2 & 5 & 6 & - \\
2 & 5 & 10 & 8 & 9 & 3 & - & 1 & 6 & - & 2 & - & - & 4 & 5 & 3 \\
10 & 8 & 7 & 6 & 1 & 2 & 5 & 6 & - & - & - & 4 & 3 & 2 & - & 1 \\
9 & 4 & 5 & 10 & 6 & 7 & - & - & - & 2 & 3 & 5 & 6 & - & 1 & 4
\end{tabular}

Fig. 4 A $5 \times 6$ triple array $T_{5}$ and its corresponding $R L$ form

Fig. 5 A Youden square produced from the $5 \times 6$ triple array $T_{5}$ in Fig. 4

$\begin{array}{rrrrrrrrrrr}\mathbf{7} & 1 & 2 & 3 & 4 & 5 & 6 & \mathbf{9} & \mathbf{1 1} & \mathbf{1 0} & \mathbf{8} \\ \mathbf{8} & 3 & \mathbf{1 1} & 4 & 1 & \mathbf{1 0} & \mathbf{9} & 2 & 5 & 6 & \mathbf{7} \\ \mathbf{9} & \mathbf{1 1} & 1 & 6 & \mathbf{1 0} & 2 & \mathbf{8} & \mathbf{7} & 4 & 5 & 3 \\ \mathbf{1 0} & 5 & 6 & \mathbf{1 1} & \mathbf{9} & \mathbf{8} & 4 & 3 & 2 & \mathbf{7} & 1 \\ \mathbf{1 1} & \mathbf{9} & \mathbf{8} & \mathbf{1 0} & 2 & 3 & 5 & 6 & \mathbf{7} & 1 & 4\end{array}$


Theorem 7 Let $T$ be a triple array with $v=r+c-1$ and $\lambda_{c c}=2$. Then $T_{R L}$ can be extended to a Youden square $Y$ that is compatible with $T$.

Proof We first note that the $R L$ form has dimensions $r \times v$, and that the basic properties of triple arrays imply that any pair of symbols in the $R L$ form occur together in $\lambda_{c c}=2$ of the columns. We use Construction 6 to produce an array $Y$. We need to verify that the array constructed in this way satisfies the basic properties of a Youden square. Properties YS2 and YS3 follow directly from Proposition 3.

We now verify that property YS1 holds, namely that every symbol appears exactly once in each row. In any pair of rows, there is exactly one column with empty cells in these rows. To see this, note that by RTA2, any two rows have exactly $\lambda_{r r}$ columns in common, and therefore, there are $v-\left(2 c-\lambda_{r r}\right)=v-(c+k)$ columns with empty cells in both rows. Since $\lambda_{c c}=2$, this equals $v-(c+k)=v-\left(c+r-\lambda_{c c}\right)=v-(c+r-2)=$ $v-(v-1)=1$.

Therefore, adding symbols according to the construction will not introduce repeated symbols in any row. Since every cell is filled, it follows that every symbol appears exactly once in each row.

By Theorem 5.2 in [3], given a $T A\left(v, k, \lambda_{r r}, 2, \lambda_{r c}: r \times c\right)$, one can construct a $(v+1, r, 2)$-SBIBD, i.e., a biplane. Taking the blocks of this SBIBD to be the columns in an array, one can then rearrange entries within columns to form a Youden square. However, this in no way guarantees that the resulting Youden square can be used in Construction 1 to give a triple array. A contribution of Theorem 7 is to give a direct way to go from the triple array $T$ to a Youden square that is compatible with $T$, i.e. that does work in Construction 1.

Note that it is not known whether there exist infinitely many biplanes, and that we have not shown that all Youden squares with parameters $(v+1, r, 2)$ will give a triple array through Construction 1 (cf. the Youden square $Y_{1}$ in Fig. 1).

\subsection{Youden squares and triple arrays with $\lambda_{c c} \geq 3$}

When attempting to use Construction 5 for $\lambda_{c c} \geq 3$, as opposed to the case when $\lambda_{c c}=2$, two new difficulties arise: First, the distribution of the empty cells is not as simple to describe. Second, the completion step is not uniquely determined. This is so because there is no longer exactly one derangement on three or more elements.

We have checked that the $R L$ forms of all triple arrays with $\lambda_{c c}=3$ given in [3] can indeed be completed to Youden squares using Construction 5. The completion in this case is very simple to carry out, and we believe that it will be possible to prove that for all triple arrays with $\lambda_{c c}=3$ and $v=r+c-1$, there exists a compatible Youden square.

For larger $\lambda_{c c}$, the completion step is more difficult since there are several possible derangements of the added symbols in a given row. Thus, there are non-trivial choices to be made at every step, and not all choices lead to a successful completion.

\section{Youden squares constructed from difference sets}

We recall some basic facts from the theory of finite fields and difference sets.

Definition 4 Let $G$ be a finite additive group of order $v$. A subset $S \subset G$ of order $k$ is a difference set with parameters $(v, k, \lambda)$ if for each non-zero $a \in G$ there are exactly $\lambda$ ordered pairs $(x, y) \in S \times S$ such that $a=x-y$. 
Proposition 4 Let $S$ be a difference set in a group $G$. Then $G \backslash S$ is also a difference set in $G$.

By applying Proposition 9.1.20 in [2], it is easy to see that the following construction produces a Youden square:

Construction 8 Let $S$ be a difference set in the additive group of $G$, with elements $0,1, \ldots, q$, and let $Y$ be an array with the columns indexed with the elements $j \in G$, and rows indexed by the elements in $S$. Write the elements of $S$ in column $C_{0}$ in arbitrary order, and then take $Y(i, j)=Y(i, 0)+j$, where $Y(i, j)$ denotes the element in position $(i, j)$ in $Y$.

Proposition 5 Let $Y$ be a Youden square constructed from a difference set $S$ in a finite group $G$ using Construction 8 , and let $R$ be the array obtained from $Y$ be removing column $C_{0}$ together with all symbols in $C_{0}$. Then $R$ satisfies condition RTA2.

Proof We must prove that any pair of rows has the same number of common occupied columns. Take two arbitrary rows $i_{1} \neq i_{2}$ in $Y$. Then by assumption $Y\left(i_{1}, 0\right)$ and $Y\left(i_{2}, 0\right)$ are both in $S$. Set $d=Y\left(i_{2}, 0\right)-Y\left(i_{1}, 0\right)$. By construction, we have $d=Y\left(i_{2}, 0\right)-Y\left(i_{1}, 0\right)=$ $Y\left(i_{2}, 0\right)+j-\left(Y\left(i_{1}, 0\right)+j\right)=Y\left(i_{2}, j\right)-Y\left(i_{1}, j\right)$ for all $j$ in $G$. Note that since $G$ is a group, there is no other way of producing this difference in $G$.

Rows $i_{1}$ and $i_{2}$ are both occupied in column $j$ iff both $I\left(i_{1}, j\right)$ and $I\left(i_{2}, j\right)$ are both in $P=G \backslash S$. Since by Proposition 4, $P$ is a difference set, the difference $d$ can be expressed as $d=p_{i}-p_{j}$ in a fixed number of ways, say $\lambda_{P}$ different ways, that is independent of $d$. It follows that any pair of rows has the same number of occupied columns in common, namely $\lambda_{P}$.

Note that since Construction 8 additively cycles through elements in $G$, the column $C_{0}$ has no privileged role. In fact, Construction 8 produces a Youden square regardless of which column is seen as the 'starting column', and Proposition 5 holds for any choice of deleted column. We will now apply Construction 8 to a specific family of difference sets. We recall some well known results.

Lemma 6 Let $q$ be an odd prime power and let $n=\frac{q-1}{2}$. Then there is a primitive element $\theta$ in $G F(q)$ that generates all non-zero elements in $G F(q)$, and $\theta^{2 n}=1$ and $\theta^{n}=-1$.

Let $Q$ denote the set of non-zero squares, let $P$ denote the set of non-squares of $G F(q)$, and let $P_{0}=P \cup\{0\}$. Then by Lemma 6,P consists of the odd powers of $\theta$ and $Q$ consists of the even powers of $\theta$. Now, $Q$ is a $\left(q, \frac{q-1}{2}, \frac{q-3}{4}\right)$ difference set in $G F(q)$, and we can construct a $\left(q, \frac{q-1}{2}, \frac{q-3}{4}\right)$-SBIBD by letting the additive group act on $Q$. We will also need the following lemma.

Lemma 7 Let $q \equiv 3(\bmod 4)$ be a prime power. Then $-1 \in P$ and $P=Q_{-}$where $Q_{-}$ denotes the set $\{-x: x \in Q\}$.

We may now prove that this particular difference set $Q$ always gives rise to a double array, but never a triple array.

Theorem 9 Let $q \equiv 3(\bmod 4)$ be a prime power, $q>3$. Then there exists a proper $D A\left(q-1, \frac{q+1}{4}, \frac{q+1}{4}, \frac{q-3}{4}: \frac{q-1}{2} \times \frac{q+1}{2}\right)$. 
Proof Let $Y$ be a $\frac{q-1}{2} \times q$ Youden square constructed from the set of squares $Q$ by Construction 8 and delete the column $C_{0}$ together with all its symbols to get a $\frac{q-1}{2} \times(q-1)$ array $R$. By Proposition 2, $R$ satisfies RTA1 and RTA3, and by Proposition $5, R$ satisfies RTA2.

We now prove that $R$ never satisfies RTA4. Fix the unique row $i_{1}$ of $R$ with $\theta^{2 n}=Y\left(i_{1}, 0\right)$. Note that for RTA4 to hold, every symbol must occur in exactly $\frac{q+1}{4}$ of the columns that have an occupied cell in this row. Now, let us consider the cells $\left(i_{1}, j\right)$ of $R$ which contain an element in $P$, that is

$$
\theta^{2 n}+j=\theta^{2 s+1}
$$

for some suitable $s \in \mathbb{Z}_{n}$. By Lemma 7 we know that the additive inverse of $\theta^{2 s+1}$ is in $Q$, so for each $s \in \mathbb{Z}_{n}$ there is an $m$ such that $\theta^{2 s+1}+\theta^{2 m}=0$. Let us check what we have in column $C_{j}$ in the row that was developed from $\theta^{2 m}$. Substitution and Lemma 6 gives

$$
\theta^{2 m}+j=-\theta^{2 s+1}+j=-\left(\theta^{2 n}+j\right)+j=-\theta^{2 n}=\theta^{n} .
$$

Hence $\theta^{n}$ occurs in all $|P|=\frac{q-1}{2}$ of these columns and since $\frac{q-1}{2}>\frac{q+1}{4}$ for all $q>3$, we have that $R$ does not satisfy RTA 4 .

Note that since $Y$ is constructed by cyclically developing a column, we have, by symmetry, that the deletion of any column together with its symbols produces the $R L$ form of a proper double array. Note also that none of the results in this section contradict Conjecture 1, since there may well be other Youden squares with the same parameters that do yield triple arrays via Construction 1. We leave it as an open problem to investigate other difference sets in this context.

\section{Paley triple arrays and Youden squares}

Our goal in this section is to show that every triple array in a certain family can be constructed from a suitable Youden square by means of Construction 1. More specifically, we will prove the following theorem.

Theorem 10 Let $q \geq 5$ be an odd prime power. Then there exists a $q \times(2 q+1)$ Youden square $Y$ that via Construction 1 gives a triple array $T$ for a suitable choice of column.

The family of triple arrays that result from the Youden squares in Theorem 10 is the family of so-called Paley triple arrays, which we will define below. We will in fact for each $q$ construct several $q \times(2 q+1)$ Youden squares that give triple arrays via Construction 1 , and we will construct a compatible Youden square for every Paley triple array.

The proof of Theorem 10 is constructive, and is structured as follows. In Sect. 5.1 we introduce Paley triple arrays and some preliminary results. In Sect. 5.2 we describe the $R L$ form of a Paley triple array, and in Sect. 5.3 how to extend this to a Youden square. In Sect. 5.4 we summarize the results from the previous sections and conclude the proof of Theorem 10.

\subsection{Construction and existence of Paley triple arrays}

We recall the following construction of Paley triple arrays given by Preece et al. [5]. Let $q$ be an odd prime power, and let $Q$ denote the set of non-zero squares of $G F(q)$ and $N$ denote the set of non-squares of $G F(q)$. Further, let $Q_{0}=Q \cup\{0\}$ and $N_{0}=N \cup\{0\}$. 
Construction 11 [5] Order the elements of $G F(q)$ as $\left\{0=w_{0}, w_{1}, \ldots, w_{q-1}\right\}$, and write $G F(q)^{\prime}=\left\{0^{\prime}=w_{0}^{\prime}, w_{1}^{\prime}, \ldots, w_{q-1}^{\prime}\right\}$, a duplicate copy. For non-zero elements $a$ and $b$ define the $q \times q$ matrix $A_{0}$ by:

$$
A_{0}(i, j)=\left\{\begin{array}{lll}
w_{i}-\frac{w_{i}-w_{j}}{a}, & \text { if } & w_{i}-w_{j} \in Q, \\
\left(w_{i}+\frac{w_{i}-w_{j}}{b}\right)^{\prime} & \text { if } & w_{i}-w_{j} \in N_{0} .
\end{array}\right.
$$

Let $A$ be the $q \times(q+1)$ matrix obtained by appending $\left(w_{0}, w_{1}, \ldots, w_{q-1}\right)$ to $A_{0}$ as column $q$, i.e. $A(i, q)=w_{i}$, for $i=0,1, \ldots, q-1$.

If an array given by Construction 11 is a triple array, then it is called a Paley triple array. Such a triple array is a $T A(2 q,(q+1) / 2,(q+1) / 2,(q-1) / 2,(q+1) / 2: q \times(q+1))$. Suitable choices for $a$ and $b$ are given in the following two theorems.

Theorem 12 [5] Suppose $q \equiv 1(\bmod 4)$. Choose $a$ and $b$ in Construction 11 such that $a b \in Q,(a-1) \in Q$ and $(b+1) \in N$. Then $A$ is a Paley triple array.

Theorem 13 [5] Suppose $q \equiv 3(\bmod 4)$. Choose $a$ and $b$ in Construction 11 such that $(a-1)(b+1) \in Q$ and if $(a-1) \in N$ then $a b \in Q$. Then $A$ is a Paley triple array.

Remark 1 In [5], the Paley triple arrays are divided into eight classes. For these classes we use the notation $T_{m, n}$, where $m$ indicates the congruence class of $q(\bmod 4)$ and $n$ indicates the type depending on the choices of $a$ and $b$, as given in the following list.

$T_{1,1}: q \equiv 1(\bmod 4), a \in Q$,

$T_{1,2}: q \equiv 1(\bmod 4), a \in N$,

$T_{3,1}: q \equiv 3(\bmod 4), a, b \in Q$ and $(a-1) \in Q$,

$T_{3,2}: q \equiv 3(\bmod 4), a, b \in Q$ and $(a-1) \in N$,

$T_{3,3}: q \equiv 3(\bmod 4), a, b \in N$ and $(a-1) \in Q$,

$T_{3,4}: q \equiv 3(\bmod 4), a, b \in N$ and $(a-1) \in N$,

$T_{3,5}: q \equiv 3(\bmod 4), a \in Q$ and $b \in N$,

$T_{3,6}: q \equiv 3(\bmod 4), a \in N$ and $b \in Q$.

Note that the defining conditions given here imply certain further conditions on $a$ and $b$ as detailed in Theorems 12 and 13.

Preece et al. [5] also proved that Paley triple arrays can be constructed for every odd prime power $q \geq 5$. In fact, the family of Paley triple arrays is the only known infinite family of triple arrays.

Lemma 8 [5]. Suppose $q \equiv 1(\bmod 4)$ is a prime power. Then there are $(q-5)(q-1) / 16$ pairs $(a, b)$ with $a \in Q$ that satisfy the conditions of Theorem 12 and there are $(q-1)^{2} / 16$ pairs $(a, b)$ with $a \in N$ that satisfy the conditions of Theorem 12. In particular, Paley triple arrays of type $T_{1,1}$ exist for every $q \geq 9$ and Paley triple arrays of type $T_{1,2}$ exist for every $q \geq 5$.

Lemma 9 [5]. Suppose $q \equiv 3(\bmod 4)$ is a prime power. Then for each of the types $T_{3,1}, T_{3,4}$ and $T_{3,5}$ there are $(q-3)^{2} / 16$ pairs $(a, b)$ that satisfy the conditions in Theorem 13, and for each of the types $T_{3,2}, T_{3,3}$ and $T_{3,6}$, there are $(q-3)(q+1) / 16$ pairs $(a, b)$ that satisfy the conditions in Theorem 13. In particular, there exist Paley triple arrays of each type $T_{3, n}$ for every $q \geq 7$ and $n=1, \ldots, 6$. 
5.2 The $R L$ form of Paley triple arrays

In order to give the $R L$ form of Paley triple arrays, we first need some preliminaries. Let $G F(q)^{*}$ denote the non-zero elements of $G F(q)$. The quadratic character $\eta: G F(q)^{*} \rightarrow$ $\{ \pm 1\}$ is defined by

$$
\eta(a)=\left\{\begin{array}{lll}
1 & \text { if } & a \in Q \\
-1 & \text { if } & a \in N .
\end{array}\right.
$$

Of Course $\eta(1)=1$, and it is also well known that

$$
\eta(-1)= \begin{cases}1 & \text { if } q \equiv 1(\bmod 4) \\ -1 & \text { if } q \equiv 3(\bmod 4) .\end{cases}
$$

Suppose $X \subset G F(q)$ and $a \in G F(q)$. We then denote by $a+X$ the set $\{a+x: x \in X\}$, and by $a X$ the set $\{a x: x \in X\}$. The following two propositions can be found in in [5].

Proposition 6 If $q \equiv 1(\bmod 4)$ then for $a \neq 0$ in $G F(q)$,

1. $\left|(a+Q) \cap Q_{0}\right|=|(a+Q) \cap N|=\left|\left(a+Q_{0}\right) \cap Q\right|=|(a+N) \cap Q|=$ $=\left|(a+N) \cap N_{0}\right|=\left|\left(a+N_{0}\right) \cap N\right|=(q-1) / 4$,

2. $\left|\left(a+Q_{0}\right) \cap N_{0}\right|=\left|\left(a+N_{0}\right) \cap Q_{0}\right|=(q+3) / 4$,

3. $\left|\left(a+Q_{0}\right) \cap N\right|=\left|(a+N) \cap Q_{0}\right|=\left|\left(a+N_{0}\right) \cap N_{0}\right|=(q-2 \eta(a)+1) / 4$,

4. $\left|(a+Q) \cap N_{0}\right|=\left|\left(a+Q_{0}\right) \cap Q_{0}\right|=\left|\left(a+N_{0}\right) \cap Q\right|=(q+2 \eta(a)+1) / 4$,

5. $|(a+Q) \cap Q|=(q-2 \eta(a)-3) / 4$,

6. $|(a+N) \cap N|=(q+2 \eta(a)-3) / 4$.

Proposition 7 If $q \equiv 3(\bmod 4)$ then for $a \neq 0$ in $G F(q)$,

1. $\left|(a+Q) \cap N_{0}\right|=\left|\left(a+Q_{0}\right) \cap Q_{0}\right|=\left|\left(a+Q_{0}\right) \cap N\right|==\left|(a+N) \cap Q_{0}\right|=\left|\left(a+N_{0}\right) \cap Q\right|=$ $\left|\left(a+N_{0}\right) \cap N_{0}\right|=(q+1) / 4$,

2. $|(a+Q) \cap Q|=|(a+N) \cap N|=(q-3) / 4$,

3. $\left|(a+Q) \cap Q_{0}\right|=|(a+N) \cap Q|=\left|\left(a+N_{0}\right) \cap N\right|=(q-2 \eta(a)-1) / 4$,

4. $|(a+Q) \cap N|=\left|\left(a+Q_{0}\right) \cap Q\right|=\left|(a+N) \cap N_{0}\right|=(q+2 \eta(a)-1) / 4$,

5. $\left|\left(a+Q_{0}\right) \cap N_{0}\right|=(q-2 \eta(a)+3) / 4$,

6. $\left|\left(a+N_{0}\right) \cap Q_{0}\right|=(q+2 \eta(a)+3) / 4$.

The following two remarks are useful when we want to determine if an element lies in $Q$ or $N$.

Remark 2 If $x_{1}, x_{2} \in Q$ and $y_{1}, y_{2} \in N$, then $x_{1} x_{2} \in Q, y_{1} y_{2} \in Q$ and $x_{1} y_{1} \in N$. This follows directly from Lemma 6 . When we consider such products in general we sometimes use the following short notation:

$$
Q Q=Q, \quad N N=Q, \quad \text { and } \quad Q N=N Q=N .
$$

Remark 3 Let $q$ be an odd prime power and let $x$ be an element in $G F(q)^{*}$. If $q \equiv 1(\bmod 4)$ then $x$ and $-x$ lie in the same set $Q$ or $N$. If $q \equiv 3(\bmod 4)$ then $x$ and $-x$ lie in different sets of $Q$ and $N$. This follows from Eq. 1 and Remark 2

The $R L$ form of Paley triple arrays can now be given directly by the following construction. 
Construction 14 Let $q$ be an odd prime power, and let $R$ be a $q \times 2 q$ array given in the following way. First index the rows by the elements of $G F(q)$ in some order $\left\{w_{0}=\right.$ $\left.0, w_{1}, w_{2}, \ldots, w_{q-1}\right\}$ and the columns by $\left\{w_{0}=0, w_{1}, \ldots, w_{q-1}, w_{0}^{\prime}=0^{\prime}, w_{1}^{\prime}, \ldots, w_{q-1}^{\prime}\right\}$ where $G F(q)^{\prime}$ is a duplicate copy of $G F(q)$. Then allocate elements of $G F(q)$ to selected cells of $R$ as follows.

$-R\left(w_{i}, w_{i}\right)=\infty$,

- if $a \in Q$ and $\left(w_{i}-w_{s}\right) \in Q$, or if $a \in N$ and $\left(w_{i}-w_{s}\right) \in N$ then

$$
R\left(w_{i}, w_{s}\right)=a\left(w_{s}-w_{i}\right)+w_{i},
$$

- if $b \in Q$ and $\left(w_{s}-w_{i}\right) \in N_{0}$, or if $b \in N$ and $\left(w_{s}-w_{i}\right) \in Q_{0}$ then

$$
R\left(w_{i}, w_{s}^{\prime}\right)=b\left(w_{i}-w_{s}\right)+w_{i} .
$$

Lemma 10 Let A be a triple array constructed by means of Construction 11 for parameters $q, a$ and $b$. Then Construction 14 with the same parameters gives its corresponding RL form $R$.

Proof The array $A$ in Construction 11 consists of triples $\left(w_{i}, w_{j}, w_{s}\right)$ given by $A\left(w_{i}, w_{j}\right)=$ $w_{s}$, (if we write $A\left(w_{i}, w_{j}\right)=A(i, j)$ ), and we can determine the corresponding $R L$ form $R\left(w_{i}, w_{s}\right)=w_{j}$ as follows. First we rename the column $q$ of Construction 11 to $\infty$, so $A\left(w_{i}, \infty\right)=w_{i}$ becomes $R\left(w_{i}, w_{i}\right)=\infty$.

First, suppose $w_{i}-w_{j} \in Q$. Then $w_{s}=w_{i}-\frac{w_{i}-w_{j}}{a}$ and $w_{j}=a\left(w_{s}-w_{i}\right)+w_{i}$. We see that $w_{i}-w_{j} \in Q$ implies $a\left(w_{i}-w_{s}\right) \in Q$, so by Remark 2, $a \in Q \Longleftrightarrow w_{i}-w_{s} \in Q$ and $a \in N \Longleftrightarrow w_{i}-w_{s} \in N$ which are the conditions on $a$.

Now suppose $w_{i}-w_{j} \in N_{0}$. Then $w_{s}=w_{i}+\frac{w_{i}-w_{j}}{b}$, and $w_{j}=b\left(w_{i}-w_{s}\right)+w_{i}$. Here $w_{i}-w_{j} \in N_{0}$ implies $b\left(w_{s}-w_{i}\right) \in N_{0}$, so by Remark $2, b \in N \Longleftrightarrow w_{s}-w_{i} \in Q_{0}$ and $b \in Q \Longleftrightarrow w_{s}-w_{i} \in N_{0}$, which are the conditions on $b$.

We rephrase Theorems 12 and 13 from [5] for the $R L$ situation.

Corollary 2 Suppose $q \equiv 1(\bmod 4)$ is a prime power. Choose $a$ and $b$ such that $a b \in$ $Q,(a-1) \in Q$ and $(b+1) \in N$. Then $R$ from Construction 14 is the RL form of a Paley triple array.

Corollary 3 Suppose $q \equiv 3(\bmod 4)$ is a prime power. Choose a and $b$ such that $(a-1)(b+$ $1) \in Q$ and if $(a-1) \in N$ then $a b \in Q$. Then $R$ from Construction 14 is the RL form of $a$ Paley triple array.

\subsection{Extension of the $R L$ form to a Youden square}

We now describe how to extend the $R L$ form from Construction 14 to a Youden square. Note that the proof that this indeed results in a Youden square is given in Sect. 5.4. First we construct an array $\bar{R}$ that will work as an extension of the array $R$ of Construction 14. That is, $R$ and $\bar{R}$ will form a Youden square when superimposed.

Construction 15 Let $R$ be the RL form constructed in Construction 14 with parameters $a, b$ and $q$. Let the array $\bar{R}$ be given by starting with an empty $q \times 2 q$ array with columns labelled by $\left\{w_{0}, w_{1}, \ldots, w_{q-1}, w_{0}^{\prime}, w_{1}^{\prime}, \ldots, w_{q-1}^{\prime}\right\}$ in this order and rows labelled by $\left\{w_{0}, w_{1}, \ldots, w_{q-1}\right\}$ in this order and performing the following steps: 
- adding the column $\infty=\left(w_{0}^{\prime}=0^{\prime}, w_{1}^{\prime}, \ldots, w_{q-1}^{\prime}\right)$ as the first column,

- for columns $\left\{w_{0}, w_{1}, \ldots, w_{q-1}\right\}$, if $a \in Q$ and $\left(w_{i}-w_{s}\right) \in N$, or if $a \in N$ and $\left(w_{i}-w_{s}\right) \in Q$, setting

$$
\bar{R}\left(w_{i}, w_{s}\right)=\left(\alpha\left(w_{s}-w_{i}\right)+w_{i}\right)^{\prime},
$$

- for columns $\left\{w_{0}^{\prime}, w_{1}^{\prime}, \ldots, w_{q-1}^{\prime}\right\}$, if $b \in Q$ and $\left(w_{s}-w_{i}\right) \in Q$, or if $b \in N$ and $\left(w_{s}-w_{i}\right) \in N$, setting

$$
\bar{R}\left(w_{i}, w_{s}^{\prime}\right)=\left(\beta\left(w_{i}-w_{s}\right)+w_{i}\right)^{\prime},
$$

where $\alpha, \beta \in G F(q)^{*}$ are chosen as follows. Suppose $q \equiv 1(\bmod 4)$, then choose $\alpha$ and $\beta$ such that $\alpha-1, \beta-1 \in Q$ and such that $\alpha$ and $\beta$ are in the same set $Q$ or $N$. Suppose $q \equiv 3(\bmod 4)$, then we have two cases:

$\star$ if $a$ and $b$ are in the same set $Q$ or $N$, then choose $\alpha$ and $\beta$ such that $\alpha-1$ and $\beta-1$ are both in one of the sets $Q$ and $N$ but $\alpha$ and $\beta$ are in different sets of $Q$ and $N$,

$\star$ if $a$ and $b$ are in different sets of $Q$ and $N$, then choose $\alpha$ and $\beta$ such that $\alpha-1, \beta-1 \in N$ and such that $\alpha$ and $\beta$ are in the same set $Q$ or $N$.

We may now detail how to produce the array $Y$, which will be proved to be a Youden square.

Remark 4 Let $R$ be an array constructed by Construction 14, satisfying the conditions of Corollary 2 or Corollary 3 , and let $\bar{R}$ be the corresponding extension of $R$ as given in Construction 15. Then we write $Y$ for the $q \times(2 q+1)$ array one gets when $R$ and $\bar{R}$ are superimposed. When superimposing $R$ and $\bar{R}$, we consider $R$ to have an empty column $\infty$, so as to coincide with $\bar{R}$ with regards to dimension.

Before we can prove that $Y$ satisfies the claims of Theorem 10 we need to prove some lemmas. We know from Lemmas 8 and 9 that for every odd prime power $q \geq 5$ it is possible to choose $a$ and $b$ that satisfy the conditions in Construction 14, but we also need to confirm that we can choose $\alpha$ and $\beta$ that satisfy the conditions in Construction 15 .

Lemma 11 Suppose $q \equiv 1(\bmod 4)$. Then in Construction 15 there are $(q-5)^{2} / 16$ pairs $(\alpha, \beta)$ with $\alpha, \beta \in Q$ and $(q-1)^{2} / 16$ pairs $(\alpha, \beta)$ with $\alpha \in N$ such that $(\alpha-1),(\beta-1) \in Q$.

Proof First, suppose that $\alpha, \beta \in Q$. Then the number of choices for $\alpha \in Q$ and $(\alpha-1) \in Q$ is given by Proposition 6 to be $|(1+Q) \cap Q|=(q-2 \eta(1)-3) / 4=(q-5) / 4$, and if $\beta \in Q$ and $(\beta-1) \in Q$, a similar argument shows that the number of choices of $\alpha \in Q$ is again $(q-5) / 4$. Thus the total number of choices of $(\alpha, \beta)$ is in this case $(q-5)^{2} / 16$.

Suppose now that $\alpha, \beta \in N$. Then the number of choices for $\alpha \in N$ and $(\alpha-1) \in Q$ is $|(1+Q) \cap N|=(q-1) / 4$, and similarly for $\beta \in N$ and $(\beta-1) \in Q$, the number of choices is again $(q-1) / 4$. The result now follows.

Lemma 12 Suppose $q \equiv 3(\bmod 4)$. If $\alpha$ and $\beta$ are in different sets $Q, N$, then in Construction 15 there are $(q-3)(q-1) / 4$ pairs $(\alpha, \beta)$ such that $(\alpha-1)$ and $(\beta-1)$ are in the same set $Q$ or $N$. If $\alpha$ and $\beta$ are in the same set $Q$ or $N$, then there are $(q-3)^{2} / 8$ pairs $(\alpha, \beta)$ such that $(\alpha-1),(\beta-1) \in N$.

Proof First we count the number of pairs $(\alpha, \beta)$ where $\alpha$ and $\beta$ are in different sets and $(\alpha-1)$ and $(\beta-1)$ are in the same set. Suppose $\alpha \in Q$ and $\beta \in N$. Then the number of choices for $(\alpha, \beta)$ where $(\alpha-1),(\beta-1) \in Q$ is $|(1+Q) \cap Q| \cdot|(1+Q) \cap N|$, and the 
number of choices where $(\alpha-1),(\beta-1) \in N$ is $|(1+N) \cap Q| \cdot|(1+N) \cap N|$. We use Proposition 7 to calculate the total number of choices for these cases:

$$
\begin{aligned}
& |(1+Q) \cap Q| \cdot|(1+Q) \cap N|+|(1+N) \cap Q| \cdot|(1+N) \cap N| \\
& =\frac{q-3}{4} \cdot \frac{q+2 \eta(1)-1}{4}+\frac{q-2 \eta(1)-1}{4} \cdot \frac{q-3}{4} \\
& =\frac{(q-3)(q-1)}{8}
\end{aligned}
$$

Letting $\alpha \in N$ and $\beta \in Q$ but keeping $(\alpha-1)$ and $(\beta-1)$ in same set gives the same number of choices. Adding these numbers gives a total of $(q-3)(q-1) / 4$ choices.

We now count the number of pairs $(\alpha, \beta)$ where $\alpha$ and $\beta$ are in the same set and $(\alpha-$ $1),(\beta-1) \in N$. If $\alpha, \beta \in Q$, then the number of choices for $(\alpha, \beta)$ is $|(1+N) \cap Q|^{2}=$ $((q-2 \eta(1)-1) / 4)^{2}=(q-3)^{2} / 16$. If $\alpha, \beta \in N$, then the number of choices is $\mid(1+N) \cap$ $\left.N\right|^{2}=(q-3)^{2} / 16$. Hence, the total number of pairs $(\alpha, \beta)$ where $(\alpha-1),(\beta-1) \in N$ and $\alpha$ and $\beta$ are in the same set is $(q-3)^{2} / 8$.

The next lemma is useful when we want to determine the support of a column of $Y$. For clarity and to save space we use a special notation in connection with this. Let $S_{w_{0}}$ be the support of column $w_{0}$. Then by construction, $S_{w_{0}}=X \cup M^{\prime}$, for some subset $X$ of $G F(q)$ and some subset $M^{\prime}$ of $G F(q)^{\prime}$. For the set $\left(X+w_{s}\right) \cup\left(M^{\prime}+w_{s}^{\prime}\right)$ we use the short notation $S_{w_{0}}+\tilde{w}_{s}$.

Lemma 13 Let $Y$ be an array as in Remark 4 and let $S_{c}$ be the support of column $c$ of $Y$. Then we have

$$
S_{w_{s}}=S_{w_{0}}+\tilde{w}_{s} \text { and } S_{w_{s}^{\prime}}=S_{w_{0}^{\prime}}+\tilde{w}_{s}
$$

respectively, for any $c \neq \infty$.

Proof For a fixed column $w_{s} \in\left\{w_{0}, w_{1}, \ldots, w_{q-1}\right\}$ we can write

$$
\begin{aligned}
S_{w_{s}}-\tilde{w}_{s} & =\left\{a\left(w_{s}-w_{i}\right)+w_{i}-w_{s}: T_{m, n}\right\} \cup\left\{\left(\alpha\left(w_{s}-w_{i}\right)+w_{i}-w_{s}: T_{m, n}\right)^{\prime}\right\} \\
& =\left\{(a-1)\left(w_{s}-w_{i}\right): T_{m, n}\right\} \cup\left\{\left((\alpha-1)\left(w_{s}-w_{i}\right): T_{m, n}\right)^{\prime}\right\}, \\
S_{w_{0}} & =\left\{a\left(0-w_{i}\right)+w_{i}: T_{m, n}\right\} \cup\left\{\left(\alpha\left(0-w_{i}\right)+w_{i}: T_{m, n}\right)^{\prime}\right\} \\
& =\left\{(1-a) w_{i}: T_{m, n}\right\} \cup\left\{\left((1-\alpha) w_{i}: T_{m, n}\right)^{\prime}\right\} .
\end{aligned}
$$

For a fixed column $w_{s}^{\prime} \in\left\{w_{0}^{\prime}, w_{1}^{\prime}, \ldots, w_{q-1}^{\prime}\right\}$ we can write

$$
\begin{aligned}
S_{w_{s}^{\prime}}-\tilde{w}_{s} & =\left\{b\left(w_{i}-w_{s}\right)+w_{i}-w_{s}: T_{m, n}\right\} \cup\left\{\left(\beta\left(w_{s}-w_{i}\right)+w_{i}-w_{s}: T_{m, n}\right)^{\prime}\right\} \\
& =\left\{(b+1)\left(w_{i}-w_{s}\right): T_{m, n}\right\} \cup\left\{\left((1-\beta)\left(w_{i}-w_{s}\right): T_{m, n}\right)^{\prime}\right\}, \\
S_{w_{0}^{\prime}} & =\left\{b\left(w_{i}-0\right)+w_{i}: T_{m, n}\right\} \cup\left\{\left(\beta\left(0-w_{i}\right)+w_{i}: T_{m, n}\right)^{\prime}\right\} \\
& =\left\{(b+1) w_{i}: T_{m, n}\right\} \cup\left\{\left((1-\beta) w_{i}: T_{m, n}\right)^{\prime}\right\} .
\end{aligned}
$$

We will show that for all types $T_{m, n}$ listed in Remark 1 the following four equations hold, which, in light of the above, will give the result.

$$
\begin{aligned}
\left\{(a-1)\left(w_{s}-w_{i}\right): T_{m, n}\right\} & =\left\{(1-a) w_{i}: T_{m, n}\right\} \\
\left\{(b+1)\left(w_{i}-w_{s}\right): T_{m, n}\right\} & =\left\{(b+1) w_{i}: T_{m, n}\right\} \\
\left\{\left((\alpha-1)\left(w_{s}-w_{i}\right): T_{m, n}\right)^{\prime}\right\} & =\left\{\left((1-\alpha) w_{i}: T_{m, n}\right)^{\prime}\right\} \\
\left\{\left((1-\beta)\left(w_{i}-w_{s}\right): T_{m, n}\right)^{\prime}\right\} & =\left\{\left((1-\beta) w_{i}: T_{m, n}\right)^{\prime}\right\}
\end{aligned}
$$


We first verify Eqs. 2 and 3. For $T_{1,1}$, Construction 14 gives that $\left(w_{i}-w_{s}\right) \in Q$, which by Remark 3 in this case means that $\left(w_{s}-w_{i}\right) \in Q$. From Corollary 2 we know that $(a-1) \in Q$, so by Remark 2 we know that the left hand side of Eq. 2 is $(a-1)\left(w_{s}-w_{i}\right)=Q$ as $w_{s}-w_{i}$ runs over all elements of $Q$. For the right hand side of Eq. 2, we again use Remark 3 to see that $(1-a) \in Q$. Construction 14 gives that $w_{i} \in Q$ and Remark 2 gives that $(1-a) w_{i}=Q$.

The results for the other types are obtained in a similar way and they are listed below. We can see that Eqs. 2 and 3 hold for them all.

\begin{tabular}{ccccc} 
& $(a-1)\left(w_{s}-w_{i}\right)(1-a) w_{i}$ & $(b+1)\left(w_{i}-w_{s}\right)$ & $(b+1) w_{i}$ \\
\hline$T_{1,1}:$ & $Q Q=Q$ & $Q Q=Q$ & $N N_{0}=Q_{0}$ & $N N_{0}=Q_{0}$ \\
$T_{1,2}:$ & $Q N=N$ & $Q N=N$ & $N Q_{0}=N_{0}$ & $N Q_{0}=N_{0}$ \\
$T_{3,1}:$ & $Q N=N$ & $N Q=N$ & $N N_{0}=Q_{0}$ & $N N_{0}=Q_{0}$ \\
$T_{3,2}:$ & $N N=Q$ & $Q Q=Q$ & $N Q_{0}=N_{0}$ & $N Q_{0}=N_{0}$ \\
$T_{3,3}:$ & $Q Q=Q$ & $N N=Q$ & $Q N_{0}=N_{0}$ & $Q N_{0}=N_{0}$ \\
$T_{3,4}:$ & $N Q=N$ & $Q N=N$ & $N N_{0}=Q_{0}$ & $N N_{0}=Q_{0}$ \\
$T_{3,5}:$ & $Q N=N$ & $N Q=N$ & $Q N_{0}=N_{0}$ & $Q N_{0}=N_{0}$ \\
$T_{3,6}:$ & $Q Q=Q$ & $N N=Q$ & $Q Q_{0}=Q_{0}$ & $Q Q_{0}=Q_{0}$
\end{tabular}

We now verify Eqs. 4 and 5 in the same manner. Here we need to look at subcases of some $T_{m, n}$ and for them we use the notation $T_{m, n, 1}$ if $(\alpha-1) \in Q$, and $T_{m, n, 2}$ if $(\alpha-1) \in N$. To emphasize that a set $X$ is a subset of $G F(q)^{\prime}$ we write $X^{\prime}$. As an illustration of the general method, we verify Eq. 5 for type $T_{3,1,2}$.

We start with the left hand side $\left((1-\beta)\left(w_{i}-w_{s}\right)\right)^{\prime}$. From Remark 1 we have that $a, b \in Q$ and by Construction 15 this implies that $(\alpha-1)$ and $(\beta-1)$ are chosen from the same set $Q$ or $N$. Since we are considering the case $T_{3,1,2}$, we have that $(\alpha-1) \in N$. We therefore know that $(\beta-1) \in N$ and then by Remark 3 that $(1-\beta) \in Q$. Since $b \in Q$, Construction 15 gives that $\left(w_{s}-w_{i}\right) \in Q$, and, again using Remark 3 , we have that $\left(w_{i}-w_{s}\right) \in N$. Hence, by Remark 2, we know that $\left((1-\beta)\left(w_{i}-w_{s}\right)\right)^{\prime}=(Q N)^{\prime}=N^{\prime}$, and in Eq. 5 it is easy to see that the right hand side will give the same result. The results for the other types are obtained in a similar way and they are listed below. We can see that Eqs. 4 and 5 hold for them all.

$\begin{array}{lcccc} & \left((\alpha-1)\left(w_{s}-w_{i}\right)\right)^{\prime} & \left((1-\alpha) w_{i}\right)^{\prime} & \left((1-\beta)\left(w_{i}-w_{s}\right)\right)^{\prime} & \left((1-\beta) w_{i}\right)^{\prime} \\ T_{1,1}: & (Q N)^{\prime}=N^{\prime} & (Q N)^{\prime}=N^{\prime} & (Q Q)^{\prime}=Q^{\prime} & (Q Q)^{\prime}=Q^{\prime} \\ T_{1,2}: & (Q Q)^{\prime}=Q^{\prime} & (Q Q)^{\prime}=Q^{\prime} & (Q N)^{\prime}=N^{\prime} & (Q N)^{\prime}=N^{\prime} \\ T_{3,1,1}: & (Q Q)^{\prime}=Q^{\prime} & (N N)^{\prime}=Q^{\prime} & (N N)^{\prime}=Q^{\prime} & (N N)^{\prime}=Q^{\prime} \\ T_{3,1,2}: & (N Q)^{\prime}=N^{\prime} & (Q N)^{\prime}=N^{\prime} & (Q N)^{\prime}=N^{\prime} & (Q N)^{\prime}=N^{\prime} \\ T_{3,2,1}: & (Q Q)^{\prime}=Q^{\prime} & (N N)^{\prime}=Q^{\prime} & (N N)^{\prime}=Q^{\prime} & (N N)^{\prime}=Q^{\prime} \\ T_{3,2,2}: & (N Q)^{\prime}=N^{\prime} & (Q N)^{\prime}=N^{\prime} & (Q N)^{\prime}=N^{\prime} & (Q N)^{\prime}=N^{\prime} \\ T_{3,3,1}: & (Q N)^{\prime}=N^{\prime} & (N Q)^{\prime}=N^{\prime} & (N Q)^{\prime}=N^{\prime} & (N Q)^{\prime}=N^{\prime} \\ T_{3,3,2}: & (N N)^{\prime}=Q^{\prime} & (Q Q)^{\prime}=Q^{\prime} & (Q Q)^{\prime}=Q^{\prime} & (Q Q)^{\prime}=Q^{\prime} \\ T_{3,4,1}: & (Q N)^{\prime}=N^{\prime} & (N Q)^{\prime}=N^{\prime} & (N Q)^{\prime}=N^{\prime} & (N Q)^{\prime}=N^{\prime} \\ T_{3,4,2}: & (N N)^{\prime}=Q^{\prime} & (Q Q)^{\prime}=Q^{\prime} & (Q Q)^{\prime}=Q^{\prime} & (Q Q)^{\prime}=Q^{\prime} \\ T_{3,5}: & (N Q)^{\prime}=N^{\prime} & (Q N)^{\prime}=N^{\prime} & (Q Q)^{\prime}=Q^{\prime} & (Q Q)^{\prime}=Q^{\prime} \\ T_{3,6}: & (N N)^{\prime}=Q^{\prime} & (Q Q)^{\prime}=Q^{\prime} & (Q N)^{\prime}=N^{\prime} & (Q N)^{\prime}=N^{\prime}\end{array}$

Using Lemma 13 and Construction 14 we can determine $S_{w_{0}}$ and $S_{w_{0}^{\prime}}$ for all types. We use the notation $Q_{\infty}=Q \cup\{\infty\}$ and $N_{\infty}=N \cup\{\infty\}$.

Corollary 4 Let $Y$ be an array as described in Remark 4. Then the supports $S_{w_{0}}$ and $S_{w_{0}^{\prime}}$ of columns $w_{0}$ and $w_{0}^{\prime}$ are given by the following list. 


$$
\begin{array}{lll}
T_{1,1}: & S_{w_{0}}=Q_{\infty} \cup N^{\prime} & S_{w_{0}^{\prime}}=Q_{0} \cup Q^{\prime} \\
T_{1,2}: & S_{w_{0}}=N_{\infty} \cup Q^{\prime} & S_{w_{0}^{\prime}}=N_{0} \cup N^{\prime} \\
T_{3,1,1}: & S_{w_{0}}=N_{\infty} \cup Q^{\prime} & S_{w_{0}^{\prime}}=Q_{0} \cup Q^{\prime} \\
T_{3,1,2}: & S_{w_{0}}=N_{\infty} \cup N^{\prime} & S_{w_{0}^{\prime}}=Q_{0} \cup N^{\prime} \\
T_{3,2,1}: & S_{w_{0}}=Q_{\infty} \cup Q^{\prime} & S_{w_{0}^{\prime}}=N_{0} \cup Q^{\prime} \\
T_{3,2,2}: & S_{w_{0}}=Q_{\infty} \cup N^{\prime} & S_{w_{0}^{\prime}}=N_{0} \cup N^{\prime} \\
T_{3,3,1}: & S_{w_{0}}=Q_{\infty} \cup N^{\prime} & S_{w_{0}^{\prime}}=N_{0} \cup N^{\prime} \\
T_{3,3,2}: & S_{w_{0}}=Q_{\infty} \cup Q^{\prime} & S_{w_{0}^{\prime}}=N_{0} \cup Q^{\prime} \\
T_{3,4,1}: & S_{w_{0}}=N_{\infty} \cup N^{\prime} & S_{w_{0}^{\prime}}=Q_{0} \cup N^{\prime} \\
T_{3,4,2}: & S_{w_{0}}=N_{\infty} \cup Q^{\prime} & S_{w_{0}^{\prime}}=Q_{0} \cup Q^{\prime} \\
T_{3,5}: & S_{w_{0}}=N_{\infty} \cup N^{\prime} & S_{w_{0}^{\prime}}=N_{0} \cup Q^{\prime} \\
T_{3,6}: & S_{w_{0}}=Q_{\infty} \cup Q^{\prime} & S_{w_{0}^{\prime}}=Q_{0} \cup N^{\prime}
\end{array}
$$

\subsection{Proof of Theorem 10}

We are now ready to prove that for each odd prime power $q \geq 5$ there exists a $(q \times(2 q+1))$ Youden square that gives a Paley triple array $T$ via Construction 1 modulo a suitably chosen column.

Proof (Proof of Theorem 10) Regarding the existence of the array $Y$, we know from Lemmas $8,9,11$ and 12 that for every odd prime power $q \geq 5$ there exist $a, b, \alpha$ and $\beta$, such that we can construct an array $Y$, that via Construction 1 , modulo column $\infty$, gives a Paley triple array. Also, by Lemma 10, for each Paley triple array, we can construct a corresponding $R$. By Corollary 2 there exists a corresponding $R$ for type $T_{1,1}$ for every $q \geq 9$, and of type $T_{1,2}$ for every $q \geq 5$, and by Corollary 3 , there exists a corresponding array $R$ for each type $T_{3, n}, n=1,2, \ldots 6$, for every $q \geq 7$.

As noted in Remark 4, $Y$ consists of $R$ and $\bar{R}$ superimposed, and $R$ is the $R L$ form of a Paley triple array. We also know that $R$ and $\bar{R}$ have disjoint sets of symbols and, in particular, that column $\infty$ of $\bar{R}$ has no symbols in common with $R$. Hence, we can use Construction 1 on $Y$ modulo column $\infty$ to produce the $R L$ form of a Paley triple array.

What is left to prove is that $Y$ is in fact a Youden square. To do this we show that each cell in the $q \times(2 q+1)$ array $Y$ is used exactly once (that is, when superimposing $R$ and $\bar{R}$, every cell contains exactly one symbol), that there are no repeated symbols in any row or column, and that each pair of columns intersects in exactly $(q-1) / 2$ symbols.

Each cell is used exactly once.

For columns $\left\{w_{0}, w_{1}, \ldots, w_{q-1}\right\}$ we have the following. If $a \in Q$, then Construction 14 fills all cells $Y\left(w_{i}, w_{s}\right)$ where $\left(w_{i}-w_{s}\right) \in Q_{0}$ and Construction 15 fills all cells $Y\left(w_{i}, w_{s}\right)$ where $\left(w_{i}-w_{s}\right) \in N$. If $a \in N$, then Construction 14 fills all cells $Y\left(w_{i}, w_{s}\right)$ where $\left(w_{i}-w_{s}\right) \in N_{0}$ and Construction 15 fills all cells $Y\left(w_{i}, w_{s}\right)$ where $\left(w_{i}-w_{s}\right) \in Q$.

For columns $\left\{w_{0}^{\prime}, w_{1}^{\prime}, \ldots, w_{q-1}^{\prime}\right\}$ we have the following. If $b \in Q$, then Construction 14 fills all cells $Y\left(w_{i}, w_{s}^{\prime}\right)$ where $\left(w_{s}-w_{i}\right) \in N_{0}$ and Construction 15 fills all cells $Y\left(w_{i}, w_{s}^{\prime}\right)$ where $\left(w_{s}-w_{i}\right) \in Q$. If $b \in N$, then Construction 14 fills all cells $Y\left(w_{i}, w_{s}^{\prime}\right)$ where $\left(w_{s}-w_{i}\right) \in Q_{0}$ and Construction 15 fills all cells $Y\left(w_{i}, w_{s}^{\prime}\right)$ where $\left(w_{s}-w_{i}\right) \in N$.

We see that in each case the two constructions complete each other as they allocate symbols to different cells. We also see that all cells are filled, as all ordered pairs $\left(w_{i}, w_{s}\right)$ are covered by the differences used. 
There are no repeated symbols in any column.

First we note that by construction, there is no repetition in column $\infty$. For a fixed $w_{s}$, the column $w_{s}$ contains symbols

$$
\left\{a\left(w_{s}-w_{i}\right)+w_{i}: T_{m, n}\right\} \cup\left\{\left(\alpha\left(w_{s}-w_{i}\right)+w_{i}: T_{m, n}\right)^{\prime}\right\},
$$

where $w_{s}$ is fixed and which of the $w_{i}$ should be used depends on the type $T_{m, n}$ as given in Constructions 14 and 15 . This can be written as

$$
\left\{a w_{s}+(1-a) w_{i}: T_{m, n}\right\} \cup\left\{\left(\alpha w_{s}+(1-\alpha) w_{i}: T_{m, n}\right)^{\prime}\right\},
$$

which gives distinct values for every $w_{i}$. Similarly, this also holds for a column $w_{s}^{\prime}$ as it contains symbols

$$
\left\{(b+1) w_{i}-b w_{s}\right\} \cup\left\{\left(\beta w_{s}+(1-\beta) w_{i}\right)^{\prime}\right\} .
$$

There are no repeated symbols in any row.

The support of a row $w_{i}$ consists of

$$
\begin{gathered}
\left\{w_{i}^{\prime}\right\} \cup\{\infty\} \cup\left\{a\left(w_{s}-w_{i}\right)+w_{i}: T_{m, n}\right\} \cup\left\{\left(\alpha\left(w_{s}-w_{i}\right)+w_{i}\right)^{\prime}: T_{m, n}\right\} \cup \\
\left\{b\left(w_{i}-w_{s}\right)+w_{i}: T_{m, n}\right\} \cup\left\{\left(\beta\left(w_{s}-w_{i}\right)+w_{i}\right)^{\prime}: T_{m, n}\right\}
\end{gathered}
$$

where $w_{i}$ is fixed and which of the $w_{s}$ should be used depends on the type $T_{m, n}$ as given in Constructions 14 and 15.

The symbols in any row of the $R L$ form $R$ of the triple array given in Construction 14 are all distinct by Lemma 10 . We can easily see that the symbol $\infty$ only occurs once in each row, but we need to check that there are no repetitions among the symbols in $\bar{R}$ given in Construction 15 , that is symbols

$$
\left\{w_{i}^{\prime}\right\} \cup\left\{\left(\alpha\left(w_{s}-w_{i}\right)+w_{i}\right)^{\prime}: T_{m, n}\right\} \cup\left\{\left(\beta\left(w_{s}-w_{i}\right)+w_{i}\right)^{\prime}: T_{m, n}\right\} .
$$

As $\alpha, \beta$ and $w_{s}-w_{i}$ are all nonzero we have that only column $\infty$ has symbol $w_{i}^{\prime}$ in row $w_{i}$. Also, for a fixed $w_{i}$, there is no repetition within columns $\left\{w_{0}, w_{1}, \ldots, w_{q-1}\right\}$ as $\alpha\left(w_{s}-w_{i}\right)+$ $w_{i}=\alpha\left(w_{z}-w_{i}\right)+w_{i}$ implies $w_{s}=w_{z}$. This is also true within columns $\left\{w_{0}^{\prime}, w_{1}^{\prime}, \ldots, w_{q-1}^{\prime}\right\}$ as $\beta\left(w_{s}-w_{i}\right)+w_{i}=\beta\left(w_{z}-w_{i}\right)+w_{i}$ implies $w_{s}=w_{z}$, but it remains to check whether a symbol in some column $w_{s} \in\left\{w_{0}, w_{1}, \ldots, w_{q-1}\right\}$ can be repeated in some column $w_{s}^{\prime} \in$ $\left\{w_{0}^{\prime}, w_{1}^{\prime}, \ldots, w_{q-1}^{\prime}\right\}$. We do this in turn for each type $T_{m, n}$.

$\left[T_{1,1}\right]$ : The contribution from Construction 15 to row $w_{i}$, with $w_{i}^{\prime}$ excluded, is

$$
\left\{\left(\alpha\left(w_{s}-w_{i}\right)+w_{i}\right)^{\prime}: w_{i}-w_{s} \in N\right\} \cup\left\{\left(\beta\left(w_{s}-w_{i}\right)+w_{i}\right)^{\prime}: w_{s}-w_{i} \in Q\right\} .
$$

where we have used Remark 3 to determine in which set $w_{s}-w_{i}$ lies. By Remark 2 this never gives the same symbol twice, since $\alpha$ and $\beta$ here are chosen from the same set $Q$ or $N$ by Construction 15 . We write this set in short notation as

$$
\left(\alpha N+w_{i}\right)^{\prime} \cup\left(\beta Q+w_{i}\right)^{\prime} .
$$

For the remaining types we go directly to the short notation.

$\left[T_{1,2}\right]: \quad \quad$ The contribution to row $w_{i}$ is $\left(\alpha Q+w_{i}\right)^{\prime} \cup\left(\beta N+w_{i}\right)^{\prime}$, which never gives the same symbol twice since $\alpha$ and $\beta$ are chosen from the same set $Q$ or $N$.

$\left[T_{3,1}\right.$ and $\left.T_{3,2}\right]$ : Here we have $\left(\alpha Q+w_{i}\right)^{\prime} \cup\left(\beta Q+w_{i}\right)^{\prime}$ and this can never give the same symbol twice, since $\alpha$ and $\beta$ lie in different sets $Q$ and $N$.

$\left[T_{3,3}\right.$ and $\left.T_{3,4}\right]$ : Here the contribution is $\left(\alpha N+w_{i}\right)^{\prime} \cup\left(\beta N+w_{i}\right)^{\prime}$ and this can never give the same symbol twice, since $\alpha$ and $\beta$ lie in different sets $Q$ and $N$. 
[T,5]: $\quad$ Here we have $\left(\alpha Q+w_{i}\right)^{\prime} \cup\left(\beta N+w_{i}\right)^{\prime}$ and this can never give the same symbol twice, since $\alpha$ and $\beta$ lie in the same set $Q$ or $N$.

$\left[T_{3,6}\right]$ : $\quad$ The contribution to row $w_{i}$ is $\left(\alpha N+w_{i}\right)^{\prime} \cup\left(\beta Q+w_{i}\right)^{\prime}$ and this can never give the same symbol twice, since $\alpha$ and $\beta$ lie in the same set $Q$ or $N$.

Each pair of columns intersects in exactly $(q-1) / 2$ symbols.

First we note that the support of column $\infty$ is $G F(q)^{\prime}$. As Construction 15 gives that all the other columns have $|Q|$ or $|N|$ of their symbols in $G F(q)^{\prime}$ we know that each one of them intersects column $\infty$ in $|Q|=|N|=(q-1) / 2$ symbols.

We now calculate the number of common symbols for all the other pairs of distinct columns, using Lemma 13, Corollary 4 and Propositions 6 or 7, as applicable depending on the congruence class of $q$. Note that the element $\infty$ in $G F(q) \cup\{\infty\}$ is taken to be invariant under addition.

$\left[T_{1,1}\right]$ : Let $w_{s}$ and $w_{z}$ be two distinct columns in $\left\{w_{0}, w_{1}, \ldots, w_{q-1}\right\}$. Then

$$
\begin{aligned}
\left|S_{w_{s}} \cap S_{w_{z}}\right| & =\left|\left(S_{w_{0}}+\tilde{w}_{s}\right) \cap\left(S_{w_{0}}+\tilde{w}_{z}\right)\right| \\
& =\left|\left(Q_{\infty} \cup N^{\prime}+\tilde{w}_{s}\right) \cap\left(Q_{\infty} \cup N^{\prime}+\tilde{w}_{z}\right)\right| \\
& =\left|\left(Q_{\infty}+w_{s}\right) \cap\left(Q_{\infty}+w_{z}\right)\right|+\left|\left(N^{\prime}+w_{s}^{\prime}\right) \cap\left(N^{\prime}+w_{z}^{\prime}\right)\right| \\
& =|\{\infty\}|+\left|\left(Q+\left(w_{s}-w_{z}\right)\right) \cap Q\right|+\left|\left(N^{\prime}+\left(w_{s}^{\prime}-w_{z}^{\prime}\right)\right) \cap N^{\prime}\right| \\
& =1+\left(q-2 \eta\left(w_{s}-w_{z}\right)-3\right) / 4+\left(q+2 \eta\left(w_{s}^{\prime}-w_{z}^{\prime}\right)-3\right) / 4 \\
& =(q-1) / 2,
\end{aligned}
$$

where in the first equality we have used Lemma 13, in the second equality, Corollary 4 , and in the fifth equality, Proposition 6. Let $w_{s}$ and $w_{z}$ be two distinct columns in $\left\{w_{0}^{\prime}, w_{1}^{\prime}, \ldots, w_{q-1}^{\prime}\right\}$. Then

$$
\begin{aligned}
\left|S_{w_{s}} \cap S_{w_{z}}\right| & =\left|\left(S_{w_{0}}+\tilde{w}_{s}\right) \cap\left(S_{w_{0}}+\tilde{w}_{z}\right)\right| \\
& =\left|\left(Q_{0} \cup Q^{\prime}+\tilde{w}_{s}\right) \cap\left(Q_{0} \cup Q^{\prime}+\tilde{w}_{z}\right)\right| \\
& =\left|\left(Q_{0}+w_{s}\right) \cap\left(Q_{0}+w_{z}\right)\right|+\left|\left(Q^{\prime}+w_{s}^{\prime}\right) \cap\left(Q^{\prime}+w_{z}^{\prime}\right)\right| \\
& =\left|\left(Q_{0}+\left(w_{s}-w_{z}\right)\right) \cap Q_{0}\right|+\left|\left(Q^{\prime}+\left(w_{s}^{\prime}-w_{z}^{\prime}\right)\right) \cap Q^{\prime}\right| \\
& =\left(q+2 \eta\left(w_{s}-w_{z}\right)+1\right) / 4+\left(q-2 \eta\left(w_{s}^{\prime}-w_{z}^{\prime}\right)-3\right) / 4 \\
& =(q-1) / 2,
\end{aligned}
$$

where in the first equality we have used Lemma 13, in the second equality, Corollary 4 , and in the fifth equality, Proposition 6 . Let $w_{s} \in\left\{w_{0}, w_{1}, \ldots, w_{q-1}\right\}$ and $w_{z} \in$ $\left\{w_{0}^{\prime}, w_{1}^{\prime}, \ldots, w_{q-1}^{\prime}\right\}$. Then

$$
\begin{aligned}
\left|\left(Q_{\infty} \cup N^{\prime}+\tilde{w}_{s}\right) \cap\left(Q_{0} \cup Q^{\prime}+\tilde{w}_{z}\right)\right| & =\left|\left(Q_{\infty}+w_{s}\right) \cap\left(Q_{0}+w_{z}\right)\right|+\left|\left(N^{\prime}+w_{s}^{\prime}\right) \cap\left(Q^{\prime}+w_{z}^{\prime}\right)\right| \\
& =\left|\left(Q+\left(w_{s}-w_{z}\right)\right) \cap Q_{0}\right|+\left|\left(N^{\prime}+\left(w_{s}^{\prime}-w_{z}^{\prime}\right)\right) \cap Q^{\prime}\right| \\
& =(q-1) / 4+(q-1) / 4 \\
& =(q-1) / 2 .
\end{aligned}
$$

where in the third equality we have used Proposition 6.

For the other types $T_{m, n}$, the calculations proceed in a very similar fashion, and we omit the details. Note again that for types $T_{m, n, 1}$, we have $\alpha-1 \in Q$, and for types $T_{m, n, 2}$, we have $\alpha-1 \in N$. This concludes the proof.

The following result makes it easy to construct $Y$ when $q$ is a prime. 


\begin{tabular}{r|rrrrrrr|rrrrrrr} 
& 0 & 1 & 2 & 3 & 4 & 5 & 6 & $0^{\prime}$ & $1^{\prime}$ & $2^{\prime}$ & $3^{\prime}$ & $4^{\prime}$ & $5^{\prime}$ & $6^{\prime}$ \\
\hline 0 & $\infty$ & - & - & 6 & - & 3 & 5 & - & - & - & - & - & - & - \\
1 & 6 & $\infty$ & - & - & 0 & - & 4 & - & - & - & - & - & - & - \\
2 & 5 & 0 & $\infty$ & - & - & 1 & - & - & - & - & - & - & - & - \\
3 & - & 6 & 1 & $\infty$ & - & - & 2 & - & - & - & - & - & - & - \\
4 & 3 & - & 0 & 2 & $\infty$ & - & - & - & - & - & - & - & - & - \\
5 & - & 4 & - & 1 & 3 & $\infty$ & - & - & - & - & - & - & - & - \\
6 & - & - & 5 & - & 2 & 4 & $\infty$ & - & - & - & - & - & - & -
\end{tabular}

Fig. 6 Second stage of producing the $R L$ form of a Paley triple array with $q=7, a=2$ and $b=3$, using Construction 14

Proposition 8 Let $Y$ be an array as described in Remark 4. If $q$ is a prime and $G F(q)$ is ordered by $\{0,1, \ldots, q-1\}$, then $Y$ has cyclic transversals modulo $q$ within $G F(q)$ and $G F(q)^{\prime}$ respectively and within columns $\{0,1, \ldots, q-1\}$ and $\left\{0^{\prime}, 1^{\prime}, \ldots,(q-1)^{\prime}\right\}$ respectively. That is

$$
Y(i+1, s+1)=Y(i, s)+\tilde{1} \text { and } Y\left(i+1,(s+1)^{\prime}\right)=Y\left(i, s^{\prime}\right)+\tilde{1},
$$

where $\tilde{1}$ denotes 1 or $1^{\prime}$ as appropriate.

Proof First we consider columns $\{0,1, \ldots, q-1\}$. If $Y(i+1, s+1) \in G F(q)$ then Construction 14 gives

$$
Y(i+1, s+1)=a(s+1-(i+1))+i+1=a(s-i)+i+1=Y(i, s)+1,
$$

and if $Y(i+1, s+1) \in G F(q)^{\prime}$, then Construction 15 gives

$$
Y(i+1, s+1)=(\alpha(s+1-(i+1))+i+1)^{\prime}=(\alpha(s-i)+i+1)^{\prime}=Y(i, s)+1^{\prime} .
$$

within columns $\left\{0^{\prime}, 1^{\prime}, \ldots,(q-1)^{\prime}\right\}$ the corresponding calculations are

$$
Y\left(i+1,(s+1)^{\prime}\right)=b(i+1-(s+1))+i+1=b(i-s)+i+1=Y\left(i, s^{\prime}\right)+1,
$$

and

$$
\begin{aligned}
Y\left(i+1,(s+1)^{\prime}\right) & =(\beta(s+1-(i+1))+i+1)^{\prime}=(\beta(s-i)+i+1)^{\prime} \\
& =Y\left(i, s^{\prime}\right)+1^{\prime} .
\end{aligned}
$$

Example 2 Let $q=7 \equiv 3(\bmod 4)$, so that $Q=\{1,2,4\}$ is the set of non-zero squares in $G F(7)=\{0,1,2,3,4,5,6\}$, and $N=\{3,5,6\}$ is the set of non-zero non-squares. We select $a=2$ and $b=3$, which is in accordance with Corollary 3. For example, $a=2 \in Q$ and $b=3 \in N$ satisfy $(a-1)(b+1)=4 \in Q$, and $a-1=1 \notin N$.

We now use Construction 14 to construct an $R L$ form $R$ using this $a$ and $b$. We start with an empty $q \times 2 q$ array with rows indexed $0,1, \ldots 6$, and columns indexed $0,1, \ldots 6,0^{\prime}, 1^{\prime}, \ldots 6^{\prime}$. In the first step, we set $R(i, i)=\infty$ for $i=0,1, \ldots 6$.

In the second step of the construction we observe that $a \in Q$, so in column $w_{s}$ in the left half of the array, we will fill cells $w_{i}$ that satisfy $\left(w_{i}-w_{s}\right) \in Q$. In column $w_{s}=0$, the cells to be filled are in rows 3,5 and 6 . We fill these cells with symbols $R(1,0)=2(0-1)+1=$ $6, R(2,0)=2(0-2)+2=5$ and $R(4,0)=2(0-4)+4=3$ respectively, where the arithmetic is in $G F(7)$. We proceed similarly for the other columns $1,2, \ldots 6$, and get the array in Fig. 6. 


\begin{tabular}{r|rrrrrrr|rrrrrrr} 
& 0 & 1 & 2 & 3 & 4 & 5 & 6 & $0^{\prime}$ & $1^{\prime}$ & $2^{\prime}$ & $3^{\prime}$ & $4^{\prime}$ & $5^{\prime}$ & $6^{\prime}$ \\
\hline 0 & $\infty$ & - & - & 6 & - & 3 & 5 & 0 & 4 & 1 & - & 2 & - & - \\
1 & 6 & $\infty$ & - & - & 0 & - & 4 & - & 1 & 5 & 2 & - & 3 & - \\
2 & 5 & 0 & $\infty$ & - & - & 1 & - & - & - & 2 & 6 & 3 & - & 4 \\
3 & - & 6 & 1 & $\infty$ & - & - & 2 & 5 & - & - & 3 & 0 & 4 & - \\
4 & 3 & - & 0 & 2 & $\infty$ & - & - & - & 6 & - & - & 4 & 1 & 5 \\
5 & - & 4 & - & 1 & 3 & $\infty$ & - & 6 & - & 0 & - & - & 5 & 2 \\
6 & - & - & 5 & - & 2 & 4 & $\infty$ & 3 & 0 & - & 1 & - & - & 6
\end{tabular}

Fig. 7 Third stage of producing the $R L$ form of a Paley triple array with $q=7, a=2$ and $b=3$, using Construction 14

Fig. 8 The corresponding Paley triple array with $q=7, a=2$ and $b=3$. Rows intersect in 4 symbols, columns intersect in 3 symbols and rows intersect columns in 4 symbols

\begin{tabular}{r|rrrrrrrr} 
& 0 & 1 & 2 & 3 & 4 & 5 & 6 & $\infty$ \\
\hline 0 & $0^{\prime}$ & $2^{\prime}$ & $4^{\prime}$ & 5 & $1^{\prime}$ & 6 & 3 & 0 \\
1 & 4 & $1^{\prime}$ & $3^{\prime}$ & $5^{\prime}$ & 6 & $2^{\prime}$ & 0 & 1 \\
2 & 1 & 5 & $2^{\prime}$ & $4^{\prime}$ & $6^{\prime}$ & 0 & $3^{\prime}$ & 2 \\
3 & $4^{\prime}$ & 2 & 6 & $3^{\prime}$ & $5^{\prime}$ & $0^{\prime}$ & 1 & 3 \\
4 & 2 & $5^{\prime}$ & 3 & 0 & $4^{\prime}$ & $6^{\prime}$ & $1^{\prime}$ & 4 \\
5 & $2^{\prime}$ & 3 & $6^{\prime}$ & 4 & 1 & $5^{\prime}$ & $0^{\prime}$ & 5 \\
6 & $1^{\prime}$ & $3^{\prime}$ & 4 & $0^{\prime}$ & 5 & 2 & $6^{\prime}$ & 6
\end{tabular}

\begin{tabular}{r|r|rrrrrrr|rrrrrrr} 
& $\infty$ & 0 & 1 & 2 & 3 & 4 & 5 & 6 & $0^{\prime}$ & $1^{\prime}$ & $2^{\prime}$ & $3^{\prime}$ & $4^{\prime}$ & $5^{\prime}$ & $6^{\prime}$ \\
\hline 0 & $0^{\prime}$ & $\infty$ & $3^{\prime}$ & $6^{\prime}$ & 6 & $5^{\prime}$ & 3 & 5 & 0 & 4 & 1 & - & 2 & - & - \\
1 & $1^{\prime}$ & 6 & $\infty$ & $4^{\prime}$ & $0^{\prime}$ & 0 & $6^{\prime}$ & 4 & - & 1 & 5 & 2 & - & 3 & - \\
2 & $2^{\prime}$ & 5 & 0 & $\infty$ & $5^{\prime}$ & $1^{\prime}$ & 1 & $0^{\prime}$ & - & - & 2 & 6 & 3 & - & 4 \\
3 & $3^{\prime}$ & $1^{\prime}$ & 6 & 1 & $\infty$ & $6^{\prime}$ & $2^{\prime}$ & 2 & 5 & - & - & 3 & 0 & 4 & - \\
4 & $4^{\prime}$ & 3 & $2^{\prime}$ & 0 & 2 & $\infty$ & $0^{\prime}$ & $3^{\prime}$ & - & 6 & - & - & 4 & 1 & 5 \\
5 & $5^{\prime}$ & $4^{\prime}$ & 4 & $3^{\prime}$ & 1 & 3 & $\infty$ & $1^{\prime}$ & 6 & - & 0 & - & - & 5 & 2 \\
6 & $6^{\prime}$ & $2^{\prime}$ & $5^{\prime}$ & 5 & $4^{\prime}$ & 2 & 4 & $\infty$ & 3 & 0 & - & 1 & - & - & 6
\end{tabular}

Fig. 9 Second step of producing a Youden square $Y$ compatible with the Paley triple array in Fig. 8, using Construction 15

The third step of producing the $R L$ form is to fill cells in the right half of the array. Since $b=3 \in N$, we will be filling cells $\left(w_{i}, w_{s}^{\prime}\right)$ satisfying $\left(w_{s}-w_{i}\right) \in Q_{0}$. In column $0^{\prime}$, the cells of interest are in rows $0,3,5$ and 6 , and we fill these cells with symbols $R\left(0,0^{\prime}\right)=$ $3(0-0)+0=0, R\left(3,0^{\prime}\right)=3(3-0)+3=5, R\left(5,0^{\prime}\right)=3(5-0)+5=6$ and $R\left(6,0^{\prime}\right)=3(6-0)+6=3$ respectively. We proceed similarly for the other columns $1^{\prime}, 2^{\prime}, \ldots 6^{\prime}$, and get the array in Fig. 7 .

This completes the construction of the $R L$ form, and the corresponding $T A(14,4,4,3,4$ : $7 \times 8$ ) Paley triple array is given in Fig. 8. Pairs of rows intersect in 4 symbols, pairs of columns intersect in 3 symbols, and each row intersects each column in 4 symbols.

We will now complete the $R L$ form $R$ in Fig. 7 to a Youden square $Y$, by means of Construction 15. Since $a \in Q$ and $b \in N$, the parameters $\alpha$ and $\beta$ must be chosen in accordance with the second case. In other words, we wish to select $\alpha$ and $\beta$ from the same set $N$ or $Q$, such that $\alpha-1 \in N$ and $\beta-1 \in N$. We choose $\alpha=4$ and $\beta=4$. Note that $\alpha$ and $\beta$ do not have to be distinct in this case.

To construct $Y$ we first add a column $\infty=\left(0^{\prime}, 1^{\prime}, \ldots, 6^{\prime}\right)$. We then proceed to fill the empty cells in the left half. Since $a \in Q$, the cells to be filled are $\left(w_{i}, w_{s}\right)$ satisfying $\left(w_{i}-w_{s}\right) \in N$, which coincides with the empty cells in the array. In column 0 , we fill the empty cells with symbols $\bar{R}(3,0)=(4(3-0)+3)^{\prime}=1^{\prime}, \bar{R}(5,0)=(4(5-0)+5)^{\prime}=4^{\prime}$ and $\bar{R}(6,0)=(4(6-0)+6)^{\prime}=2^{\prime}$, respectively, where the arithmetic is in $G F(q)^{\prime}$. We proceed similarly for the other columns $1,2, \ldots, 6$, and get the array in Fig. 9. 


\begin{tabular}{r|r|rrrrrrr|rrrrrrr} 
& $\infty$ & 0 & 1 & 2 & 3 & 4 & 5 & 6 & $0^{\prime}$ & $1^{\prime}$ & $2^{\prime}$ & $3^{\prime}$ & $4^{\prime}$ & $5^{\prime}$ & $6^{\prime}$ \\
\hline 0 & $0^{\prime}$ & $\infty$ & $3^{\prime}$ & $6^{\prime}$ & 6 & $5^{\prime}$ & 3 & 5 & 0 & 4 & 1 & $2^{\prime}$ & 2 & $1^{\prime}$ & $4^{\prime}$ \\
1 & $1^{\prime}$ & 6 & $\infty$ & $4^{\prime}$ & $0^{\prime}$ & 0 & $6^{\prime}$ & 4 & $5^{\prime}$ & 1 & 5 & 2 & $3^{\prime}$ & 3 & $2^{\prime}$ \\
2 & $2^{\prime}$ & 5 & 0 & $\infty$ & $5^{\prime}$ & $1^{\prime}$ & 1 & $0^{\prime}$ & $3^{\prime}$ & $6^{\prime}$ & 2 & 6 & 3 & $4^{\prime}$ & 4 \\
3 & $3^{\prime}$ & $1^{\prime}$ & 6 & 1 & $\infty$ & $6^{\prime}$ & $2^{\prime}$ & 2 & 5 & $4^{\prime}$ & $0^{\prime}$ & 3 & 0 & 4 & $5^{\prime}$ \\
4 & $4^{\prime}$ & 3 & $2^{\prime}$ & 0 & 2 & $\infty$ & $0^{\prime}$ & $3^{\prime}$ & $6^{\prime}$ & 6 & $5^{\prime}$ & $1^{\prime}$ & 4 & 1 & 5 \\
5 & $5^{\prime}$ & $4^{\prime}$ & 4 & $3^{\prime}$ & 1 & 3 & $\infty$ & $1^{\prime}$ & 6 & $0^{\prime}$ & 0 & $6^{\prime}$ & $2^{\prime}$ & 5 & 2 \\
6 & $6^{\prime}$ & $2^{\prime}$ & $5^{\prime}$ & 5 & $4^{\prime}$ & 2 & 4 & $\infty$ & 3 & 0 & $1^{\prime}$ & 1 & $0^{\prime}$ & $3^{\prime}$ & 6
\end{tabular}

Fig. 10 A Youden square $Y$ compatible with the Paley triple array in Fig. 8, constructed using Construction 15

Finally, we fill the empty cells of the right half of the array. Since $b \in N$, the cells to be filled are $\left(w_{i}, w_{s}\right)$ satisfying $\left(w_{s}-w_{i}\right) \in N$, which again coincides with the empty cells in the array. In column $0^{\prime}$, we fill the empty cells with symbols $\bar{R}\left(1,0^{\prime}\right)=(4(1-0)+1)^{\prime}=$ $5^{\prime}, \bar{R}\left(2,0^{\prime}\right)=(4(2-0)+2)^{\prime}=3^{\prime}$ and $\bar{R}\left(4,0^{\prime}\right)=(4(4-0)+4)^{\prime}=6^{\prime}$ respectively. We proceed similarly for the other columns $1^{\prime}, 2^{\prime}, \ldots, 6^{\prime}$, and get the array in Fig. 10.

\section{Concluding remarks}

In conclusion, we pose the following problems.

Problem 1 Let $Y$ be a Youden square constructed from a difference set. Will $Y$ always give the $R L$ form $R$ of a proper double array through Construction 1 modulo any column, that is will $R$ ever satisfy condition RTA4?

Problem 2 Let $\mathcal{Y}$ be some family of Youden squares. Investigate the properties of arrays obtained from $Y \in \mathcal{Y}$ through Construction 1.

We also restate Conjecture 1 in a more general formulation.

Conjecture 2 Let $T$ be a triple array with $\lambda_{c c} \geq 2$. Then there exists a Youden square $Y$ such that $Y$ will produce the $R L$ form of $T$ through Construction 1, for some suitably chosen column.

\section{References}

1. Agrawal H.: Some methods of construction of designs for two-way elimination of heterogeneity. J. Am. Stat. Assoc. 61, 1153-1171 (1966).

2. Ionin Y.J., Shrikhande M.S.: Combinatorics of Symmetric Designs. Cambridge University Press, New York, ISBN 978-0-521-81833-9 (2006).

3. McSorley J.P., Phillips N.C.K., Wallis W.D., Yucas J.L.: Double arrays, triple arrays and balanced grids. Des. Codes Cryptogr. 35, 21-45 (2005).

4. McSorley J.P.: Double arrays, triple arrays and balanced grids with $v=r+c-1$. Des. Codes Cryptogr. 37, 313-318 (2005).

5. Preece D.A., Wallis W.D., Yucas J.L.: Paley triple arrays. Australas. J. Comb. 33, 237-246 (2005).

6. Raghavarao D., Nageswararao G.: A note on a method of construction of designs for two-way elimination of heterogeneity. Commun. Stat. 3, 197-199 (1974).

7. Wallis W.D., Yucas J.L.: Note on Agrawal's "designs for two-way elimination of heterogeneity". J. Comb. Math. Comb. Comput. 46, 155-160 (2003). 